\title{
Nanoimprint Lithography: Methods and Material Requirements**
}

\author{
By L. Jay Guo*
}

Nanoimprint lithography (NIL) is a nonconventional lithographic technique for high-throughput patterning of polymer nanostructures at great precision and at low costs. Unlike traditional lithographic ap-

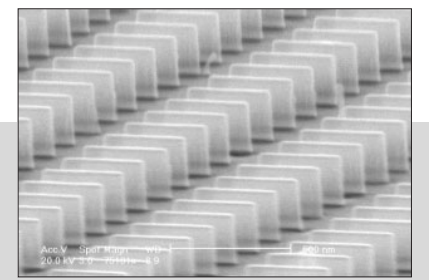
proaches, which achieve pattern definition through the use of photons or electrons to modify the chemical and physical properties of the resist, NIL relies on direct mechanical deformation of the resist material and can therefore achieve resolutions beyond the limitations set by light diffraction or beam scattering that are encountered in conventional techniques. This Review covers the basic principles of nanoimprinting, with an emphasis on the requirements on materials for the imprinting mold, surface properties, and resist materials for successful and reliable nanostructure replication.

\section{Introduction}

The ability to fabricate structures from the micro- to the nanoscale with high precision in a wide variety of materials is of crucial importance to the advancement of micro- and nanotechnology and the nanosciences. The semiconductor industry has been pushing high-precision nanoscale lithography to manufacture ever-smaller transistors and higher-density integrated circuits (ICs). Critical issues, such as resolution, reliability, speed, and overlay accuracy, all need to be addressed in order to develop new lithography methodologies for such demanding, industrially relevant processes. On the other hand, less stringent conditions are found in many other areas, for example, photonics, micro- and nanofluidics, chip-based sensors, and most biological applications. Several alternative ap-

[*] Prof. L. J. Guo

Department of Electrical Engineering and Computer Science The University of Michigan

1301 Beal Ave., Ann Arbor, MI 48109-2122 (USA)

E-mail:guo@umich.edu

[**] It is a great pleasure to acknowledge many wonderful students, postdoctoral fellows, and colleagues who have contributed to the work described in this article. This review is dedicated to all of them. Special thanks to Dr. Peng-Fei Fu at Dow Corning Corporation (USA) for the recent collaborations on the development of new materials for nanoimprint technology. This work was supported by NSF grants ECS-0424204 and ECS-0508252, AFOSR grant FA955004-1-0312, and NSFC grant No. 60528003. proaches towards nanostructure fabrication have been exploited in the past 15 years, without resorting to expensive tools such as those used in deep-UV projection lithography and electron-beam lithography. These techniques include microcontact printing (or soft lithography), ${ }^{[1]}$ nanoimprint lithography (NIL), ${ }^{[2]}$ scanning-probe-based techniques (e.g., atomic force microscope lithography), ${ }^{[3]}$ and dip-pen lithography. ${ }^{[4]}$ A good overview of several of these techniques was presented in a recent paper. ${ }^{[5]}$ This article will focus on NIL technology and review the progress that has been made in this field in recent years. Nanoimprinting can not only create resist patterns, as in lithography, but can also imprint functional device structures in various polymers, which can lead to a wide range of applications in electronics, photonics, data storage, and biotechnology. Some of these applications have been discussed in a previous review. ${ }^{[6]}$

The principle of nanoimprinting is very simple. Figure 1a shows a schematic of the originally proposed NIL process. ${ }^{[2,7]}$ A hard mold that contains nanoscale surface-relief features is pressed into a polymeric material cast on a substrate at a controlled temperature and pressure, thereby creating a thickness contrast in the polymeric material. A thin residual layer of polymeric material is intentionally left underneath the mold protrusions, and acts as a soft cushioning layer that prevents direct impact of the hard mold on the substrate and effectively protects the delicate nanoscale features on the mold surface. For most applications, this residual layer needs to be removed by an anisotropic $\mathrm{O}_{2}$ plasma-etching process to complete the pattern definition. 
a)

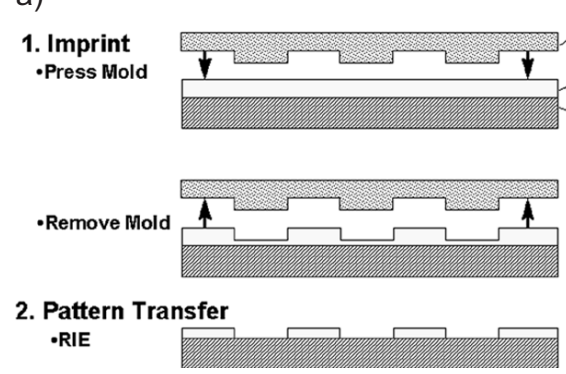

b)

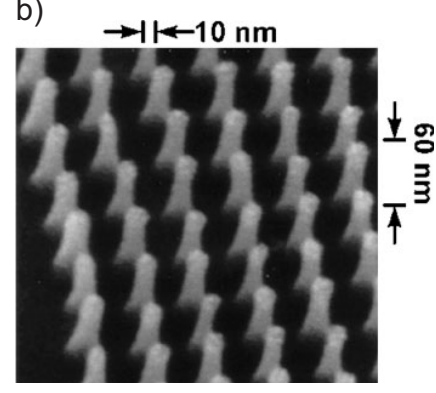

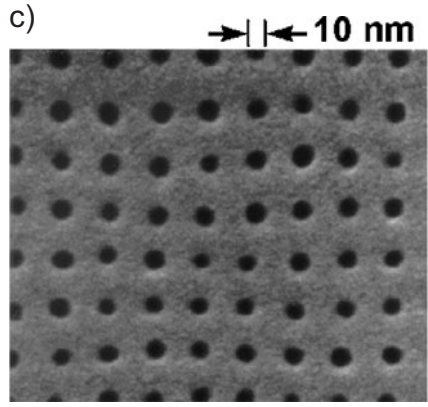

Figure 1. a) Schematic of the originally proposed NIL process. b) Scanning electron microscopy (SEM) image of a fabricated mold with a $10 \mathrm{~nm}$ diameter array. c) SEM image of hole arrays imprinted in poly (methyl methacrylate) by using such a mold. Reproduced with permission from [8]. Copyright 1997 American Institute of Physics.

What makes NIL an attractive and widely researched technology is that it demonstrated ultrahigh resolutions soon after its inception. Figure $1 \mathrm{~b}$ and $\mathrm{c}$ shows scanning electron microscopy (SEM) images of a mold with a pillar array (pillar diameter $10 \mathrm{~nm}$ ) and an imprinted $10 \mathrm{~nm}$ hole array in poly(methyl methacrylate) (PMMA) that were obtained almost a decade ago. ${ }^{[8]}$ NIL is inherently high-throughput, because of parallel printing, and it requires only a simple equipment set-up, leading to low-cost processes. A variation of the NIL technique that uses a transparent mold and UV-curable precursor liquid to define the pattern (step-and-flash imprint lithography (SFIL), Fig. 2a) was developed soon after, ${ }^{[9,10]}$ allowing the process to be carried out at room temperature and making it very attractive for IC semiconductor device manufacturers. ${ }^{[11]}$

Because of its fast development in the past decade and its potential for sub-50 nm lithography, the Massachusetts Institute of Technology's "Technology Review" listed NIL as one of 10 emerging technologies that will strongly impact the world. ${ }^{[12]}$ In 2003 the International Technology Roadmap for Semiconductors (ITRS) also announced the inclusion of NIL onto their roadmap as a candidate technology for future IC production. Significant efforts from both academia and industry have been put in SFIL research and development ${ }^{[9]}$ template-fabrication methods, ${ }^{[13,14]}$ and defect analysis. ${ }^{[14,15]}$ Figure $2 \mathrm{~b}$ and $\mathrm{c}$ shows $20 \mathrm{~nm}$ lines printed by SFIL and $40 \mathrm{~nm}$ lines printed with a template used over 1500 times, respective- ly. An overlay accuracy of $50 \mathrm{~nm}$ has been achieved by using interferometric in situ alignment techniques. ${ }^{[16]}$ Higher degrees of accuracy can also be anticipated.

Although NIL-based approaches have proven excellent resolutions, there are still significant challenges in meeting the stringent requirements of semiconductor IC manufacturing, especially in terms of defect control and production-level throughput, which requires printing 60-80 wafers per hour with extremely high yields. On the other hand, because of its simplicity this technique has found numerous applications in electronics (e.g., hybrid plastic electronics, ${ }^{[17]}$ organic electronics and photonics, ${ }^{[18,19]}$ nanoelectronic devices in $\mathrm{Si}^{[20,21]}$ and in $\mathrm{GaAs}^{[22]}$ ), in photonics (e.g., organic lasers, ${ }^{[23]}$ conjugat$\mathrm{ed}^{[24]}$ and nonlinear optical polymer nanostructures, ${ }^{[25]}$ highresolution organic light-emitting diode (OLED) pixels, ${ }^{[26,27]}$ diffractive optical elements, ${ }^{[28]}$ broadband polarizers $\left.{ }^{[29-31]}\right)$, in magnetic devices (e.g., single-domain magnetic structures, ${ }^{[32,33]}$ high-density patterned magnetic media and highcapacity disks, ${ }^{[34,35,36]}$, in nanoscale control of polymer crystallization, ${ }^{[37]}$ and in biological applications (e.g., manipulating DNA in nanofluidic channels, ${ }^{[38,39]}$ nanoscale protein patterning, ${ }^{[40,41]}$ the effect of imprinted nanostructures on cell culture $\left.^{[42]}\right)$.

Because nanoimprint lithography is based on the mechanical molding of polymer materials, which is drastically different from other traditional lithographic techniques, it faces a new set of issues and challenges. In the following, a detailed discus-

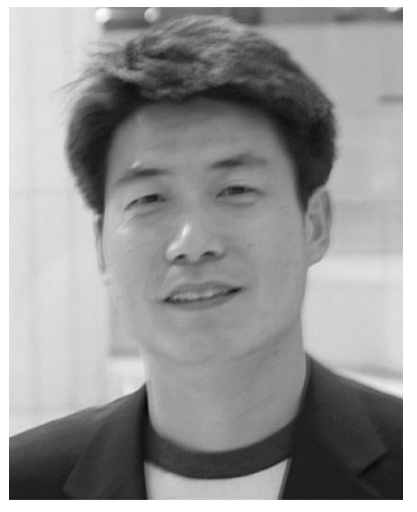

L. Jay Guo is an associate professor of Electrical Engineering and Computer Science at the University of Michigan. He has been involved in the development of nanoimprint technology since 1996 and has exploited numerous applications in electronic and photonic devices. He obtained his B.S. degree from Nankai University (China) in 1990, and M.S. and Ph.D. degrees from the University of Minnesota (USA) in 1995 and 1997, respectively. His Ph.D. thesis was on semiconductor single-electron transistors and memories. From 1997 to 1999, he was a research associate fellow at Princeton University (USA). His current research interests include the development and applications of imprinting and printing techniques to the fabrication of functional nanostructures, the study of plasmonic nanophotonics and photonic microresonators for biosensing applications, research on organic LEDs and photovotaics, and the study of nanofluidic devices. His earlier work on room-temperature operation of silicon single-electron memory was cited by the American Physical Society as one of the breakthroughs in Nanotechnology in 1997. 


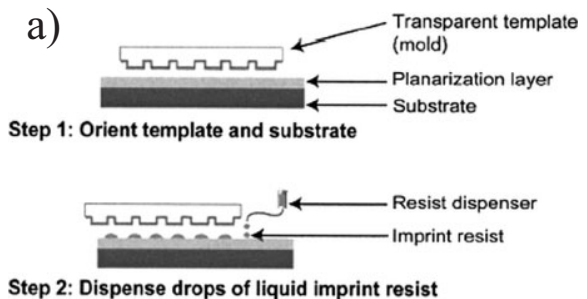

Step 2: Dispense drops of liquid imprint resist

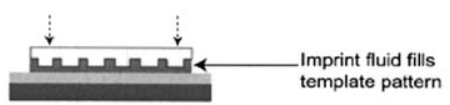

Step 3: Lower template and fill pattern

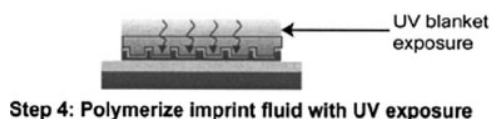

Step 4: Polymerize imprint fluid with UV exposure

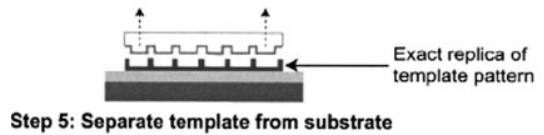

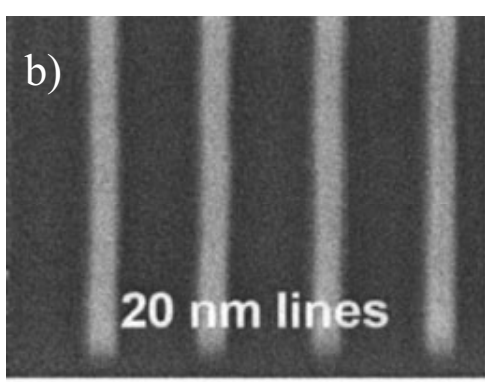

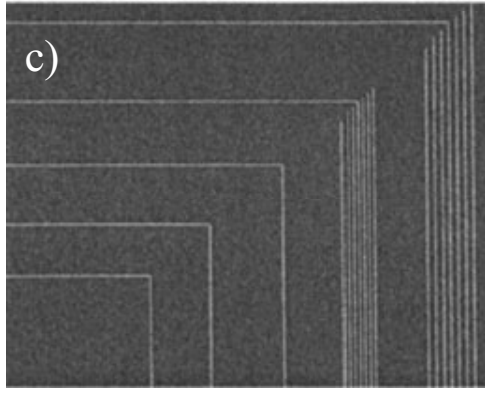

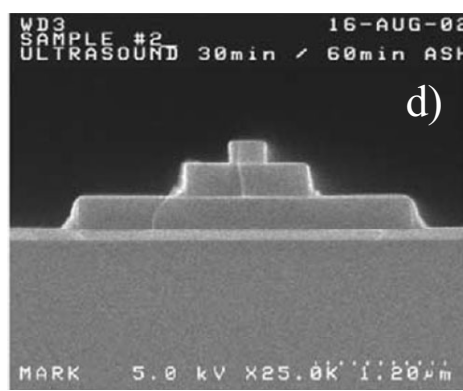

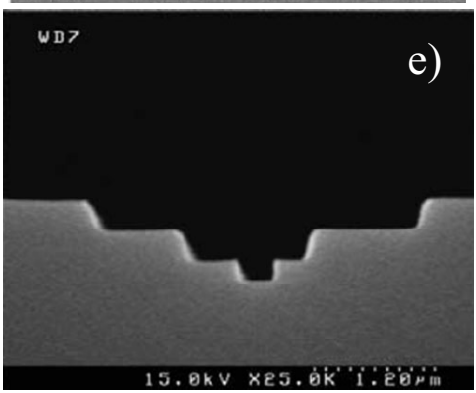

Figure 2. a) Schematic outline of the SFIL process. b) Pattern of $20 \mathrm{~nm}$ lines patterned by using SFIL. c) Pattern of $40 \mathrm{~nm}$ lines printed with a template used over 1500 times. d,e) Multitiered SFIL template and the resulting imprint in a resist material. Reproduced with permission from [10]. Copyright 2005 International Society of Optical Engineering.

sion of the requirements on the mold and resist will be presented. Critical issues in the imprinting, separation, and etching steps will be identified, and solutions to overcome the various technical challenges will be described. The effectiveness of these approaches in making NIL a successful technology will be illustrated by providing ample examples.

\section{NIL Mold}

The elements required for NIL are (i) a mold with predefined surface relief nanostructures, and (ii) a suitable resist material that can be deformed and hardened to preserve the shape of the impression. Usually, the resist material is applied on top of a substrate. The mold used in NIL can essentially be any type of solid material that has a high strength and durability. The resist material can be a thermal plastic, thermal setting, or low-viscosity precursor that can be cured either thermally or by UV light. The molds or stamps are normally made in silicon, dielectric materials (e.g, silicon dioxide or silicon nitride), metals (e.g., nickel), or polymeric materials that have a sufficient Young modulus. Common features of these different molds are that they are hard and have a high mechanical strength. The properties of these hard molds contrast those of elastomeric stamps used in soft lithography, and are essential for producing nanoscale features because the protrusion patterns on the mold should not deform, buckle, or collapse during imprinting, even at elevated temperatures. In this manner, preservation of the shape and aspect ratio as well as faithful pattern definition at size scales of $10 \mathrm{~nm}$ and below is guaranteed. These requirements do not exclude the use of a flexible backplane supporting the hard surface relief structure. It is actually advantageous for the mold to have global flexibility and local rigidity in many applications, especially when the substrate is not flat, because a flexible mold can provide large-area conformal contact with the substrate without resorting to high pressures. The rigiflex-mold approach explicitly exploits this feature for NIL patterning. ${ }^{[43]}$

\subsection{Mold Fabrication}

Considerations for selecting mold materials include hardness, compatibility with traditional microfabrication processing or the intended applications, and the thermal expansion coefficient of the material, to name an important few. Candidate materials include $\mathrm{Si}, \mathrm{SiO}_{2}, \mathrm{SiC}$, silicon nitride, metals, sapphire, and diamond film. Work from many groups has shown that $\mathrm{Si}$ and $\mathrm{SiO}_{2}$ have sufficient hardness and durability properties for nanoimprint applications. The thermal expansion coefficient is especially important in the NIL process, where a temperature of more than $100{ }^{\circ} \mathrm{C}$ is typically required at the imprinting step. A thermal mismatch between the mold and the substrate could result in pattern distortions or stress build-up during the cooling cycle, which would affect the pattern fidelity and registration accuracy. In this regard, a Si mold together with a Si substrate makes a good pair for the NIL process in applications that demand precise, critical dimensions and overlay control. The thermal expansion mismatch can be ignored if the NIL process is carried out at room temperature, for example, in UV-assisted NIL, roomtemperature NIL, ${ }^{[44]}$ or SFIL processes. 
The fabrication of the Si mold is straightforward if one has access to other lithographic processing and reactive ion etching (RIE) facilities. The commonly used processing steps are illustrated in Figure 3a. First, a resist material is spin-coated onto the mold surface (either a pure Si substrate or Si with a thermally grown oxide), followed by lithography to define the

a)

Pattern
polymer
template

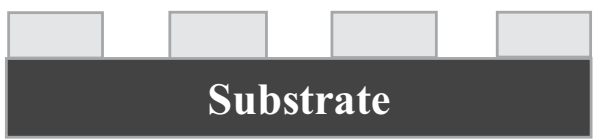

Deposit metal mask

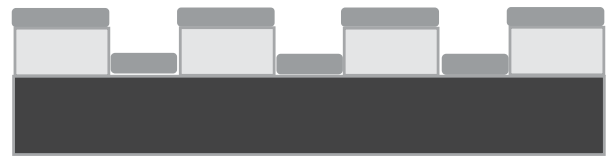

Lift-off

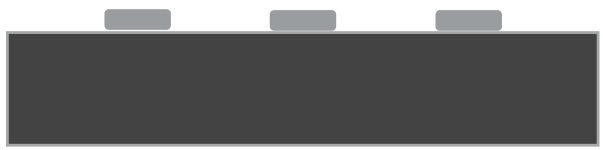

Reactive ion etching

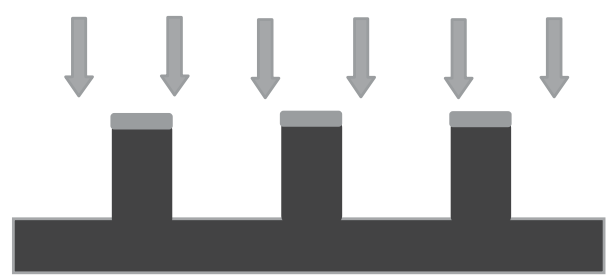

b)

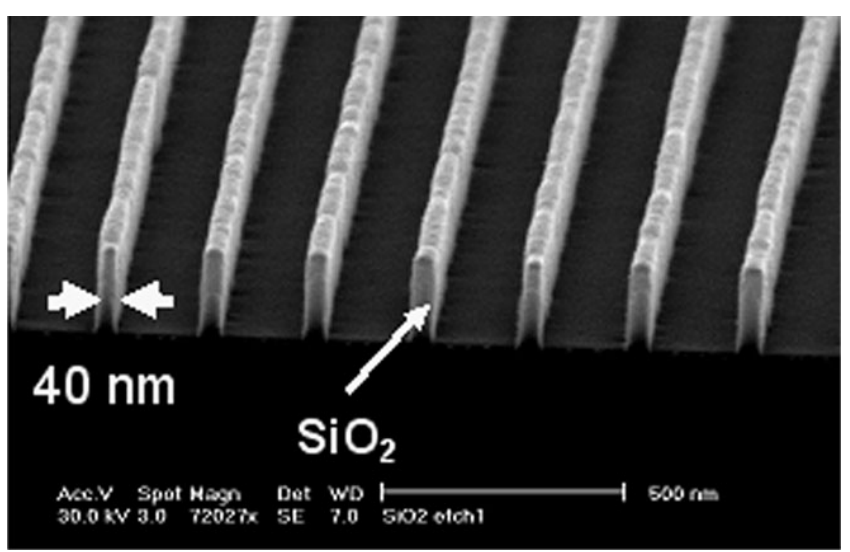

Figure 3. a) Schematic illustration of the Si mold-fabrication process. b) SEM image of a mold fabricated using thermally grown $\mathrm{SiO}_{2}$ on a substrate, with equal line widths and spacings; scale bar: $500 \mathrm{~nm}$. (The roughness seen on top of the etched oxide originates from the metal mask used in the reactive-ion-etching process that has not yet been removed). desired mold pattern. For the lithography step, one can choose from UV lithography for microscale and larger features, electron-beam lithography for very small features, interference lithography for large-area periodic features, or NIL itself. A hard masking layer, such as a metal, can be deposited over the patterned resist template, followed by a lift-off process that removes the resist template and the material on top, leaving a patterned mask layer on the Si substrate. Next, an anisotropic RIE process is used to selectively etch away the $\mathrm{Si}$ material in the unmasked region, producing the surface relief structures required for NIL. Figure $3 b$ shows an SEM image of a fabricated Si mold with protrusion features etched into a thermal oxide layer grown on top of a Si substrate. Figure 4 shows other examples where molds with different periodical
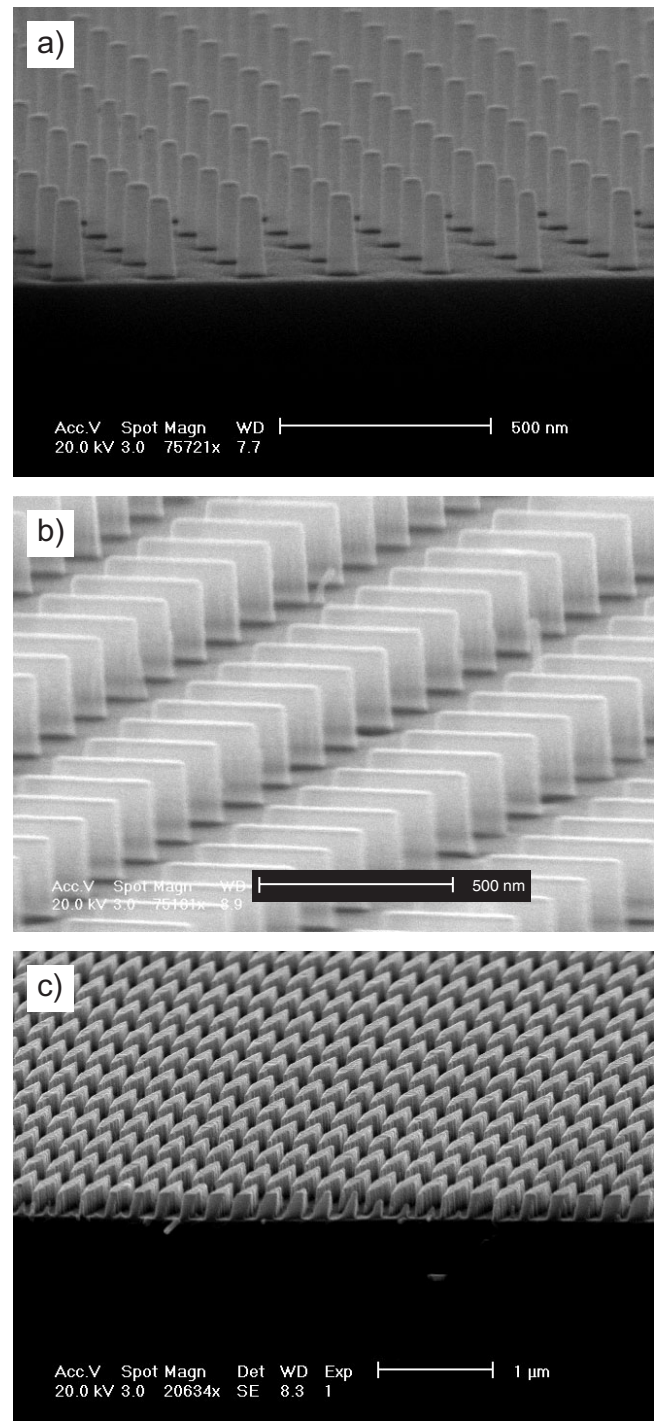

Figure 4. SEM images of large-area molds with arrays of different features a) A pillar array, produced by imprinting twice with the same grating mold but orthogonal directions. b) A bar array, produced by two grating molds with different periods; scale bar: $500 \mathrm{~nm}$. c) Diamond-shaped array, produced by two imprints that use the same grating mold but are oriented at an angle of $60^{\circ}$. 
features were fabricated by using a grating mold and performing NIL twice, at orientations of either $90^{\circ}$ or $60^{\circ}$ with respect to each other, followed by metal deposition, lift-off, and finally RIE to produce the desired mold features. These type of molds can cover large areas (several square centimeters) because the initial grating mold is fabricated by holographic interference lithography. These molds have been used in our group to fabricate uniform and oriented metal nanoparticle arrays for studying localized surface plasmon resonances. $^{[45]}$

Molds or templates for SFIL can be fabricated by similar methods. However, because SFIL requires a transparent template (typically quartz or silica) a charging effect could occur during the electron-beam lithography process, because of the nonconductive nature of these dielectric materials. This charging effect can severely distort the electron beam and affect the pattern resolution and fidelity. Several methods have been used to eliminate this effect. One method involves coating a thin metal layer (e.g., $15 \mathrm{~nm} \mathrm{Cr}$ ) on top of the quartz substrate before spin-coating the e-beam resist to help discharge the electrons. ${ }^{[13]}$ An advantage of this approach is that the $\mathrm{Cr}$ layer can be used directly as an etch mask in the subsequent RIE step to form features in the quartz template. A second method is to deposit a transparent conductive oxide layer, such as indium tin oxide (ITO), to function as both a discharge layer and as an integral part of the template structure itself. ${ }^{[14]}$ The charging effect is then not only suppressed in the e-beam lithography step during template fabrication but also at the final inspection stage by SEM, which greatly facilitates the manufacturing of the mold and the template. These two methods have been used to fabricate SFIL templates on standard photomask plates. The second method offers an additional advantage, in that multiple layers of ITO can be deposited to fabricate a 3D tiered structure. An example is shown in Figure $2 \mathrm{~d}$, showing a three-layer template structure. Figure 2e shows the imprinted pattern obtained after using such a mold. This approach can truly take advantage of the mechanical molding nature of the NIL process, and can produce 3D patterns in polymers in a single step.

Ultrahigh-resolution NIL has been demonstrated by Austin et al. through the use of a specially prepared mold, consisting of a superlattice structure grown on a GaAs substrate by molecular beam epitaxy. After the sample is cleaved, the exposed side is immersed in a chemical etchant that selectively etches one component, creating a relief structure on the sample edge. By using this special mold the authors demonstrated the imprinting of $14 \mathrm{~nm}$ pitch patterns. ${ }^{[46]}$ Tormen et al. presented interesting results by using a technique that allows the production of tightly controlled nano- to microscale 3D profiles. ${ }^{[47]}$ Finally, it is also possible to use the focused ion beam (FIB) technique to directly "carve out" the desired mold features, even 3D or gray-scale patterns that are very difficult to fabricate, or would involve multiple steps to obtain, by other lithographic techniques. An example of such a structure fabricated by FIB is shown in Figure 5. FIB may find many uses in NIL-related applications in the future.

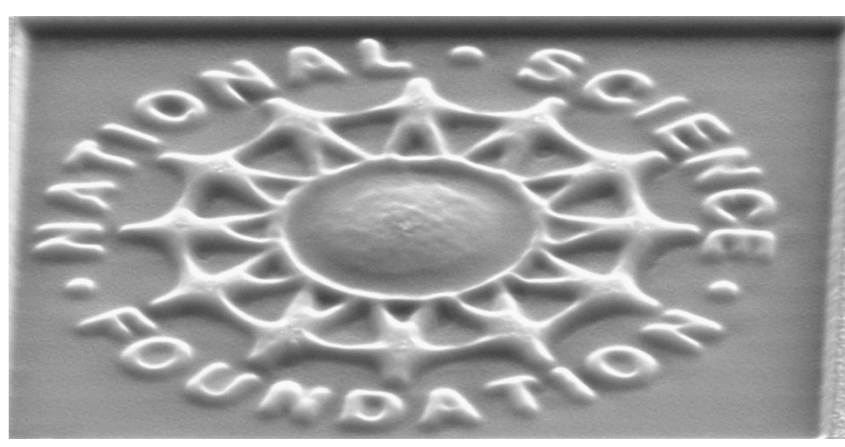

Figure 5. Mold fabricated by using FIB, showing a gray-scale pattern (an NSF logo).

\subsection{Mold Surface Preparation}

A mold for imprint lithography typically has a high density of nanoscale protrusion features on its surface. This effectively increases the total surface area that contacts the imprinted polymer, leading to strong adhesion of the imprinted polymer to the mold. This effect can easily be seen by the sticking of a resist material to a mold without any special treatment. Solutions to this problem are (i) incorporating an internal release agent into the resist formulation (as is done in the precursor mix used for SFIL), (ii) applying a low-surfaceenergy coating to the mold to reduce its surface energy (or a combination of both approaches), and (iii) choosing a mold material with an intrinsically low surface energy. The most widely adopted approach is to form a self-assembled monolayer of a fluorosilane release agent on the mold surface (e.g., $1 H, 1 H, 2 H, 2 H$-perfluorodecyl-trichlorosilane), either by a solution-phase or a vapor-phase reaction. This approach can be readily applied to oxide surfaces, or to Si surfaces that have been oxidized with a piranha soak to generate the required terminal hydroxyl groups. Jung et al. compared the substrates treated by the two different processes by atomic force microscopy (AFM), ellipsometry, IR spectroscopy, and contact-angle measurement, and concluded that the vapor-phase coating method provided superior surface-release properties. ${ }^{[48]} \mathrm{Re}-$ cently, Schift et al. found that the antiadhesion properties of mold surfaces coated with fluorinated trichlorosilanes can be further improved by co-deposition of monochlorosilanes. ${ }^{[49]}$ This is because the introduction of monochlorosilanes helps to reduce the steric hindrance between the trichlorosilane molecules bound to the mold surface, resulting in a better molecular packing compared to coatings that use only trichlorosilane molecules. It has been shown experimentally that fluorosilane-treated molds can be used to imprint several hundreds to a thousand times before their antiadhesion properties degrade and a new coating is required.

\subsection{Flexible Fluoropolymer Mold}

The durability issue of the surface coating on a mold can be alleviated if the mold itself is made from a material that has a 
low surface energy and sufficient mechanical strength. Lee et al. have demonstrated that molds made from amorphous fluoropolymers, such as Teflon AF 2400 (glass transition temperature $\left(T_{\mathrm{g}}\right) 240^{\circ} \mathrm{C}$ ), can be used as mold without any surface treatment. Teflon AF 2400 is a copolymer of 2,2-bistrifluoromethyl-4,5-difluoro-1,3-dioxole and tetrafluoroethylene. ${ }^{[50]}$ The polymer has a tensile modulus of ca. $1.6 \mathrm{GPa}$ (almost a thousand times harder than the elastomeric PDMS material), which is stiff enough for patterning small features without mold deformation. Also, the inert nature with a low surface energy of $15.6 \mathrm{dyn} \mathrm{cm}^{-1}$ (cf. PDMS, ca. $19.6 \mathrm{dyn} \mathrm{cm}^{-1}$ ) makes it easy to demold after the imprinting process without any surface treatment and without deterioration in surface properties over many imprinting cycles.

Mold fabrication can also be simplified by casting the fluoropolymer solutions over a prefabricated template and drying off the solvent ${ }^{[51]}$ or by direct molding or imprinting of the fluoropolymer at $350^{\circ} \mathrm{C}$ under high pressure. ${ }^{[52]} \mathrm{A}$ flexible film can provide a better conformal contact with the substrate to be patterned and reduce the pressure needed during the imprinting step. A further benefit is that de-molding can be achieved by pealing the mold from the imprinted substrate with an effectively smaller de-molding area. This is much easier than the de-molding of a stiff mold, where the hard mold needs to be separated from the substrate as a whole. Figure 6a shows patterned high-aspect-ratio features obtained by using a Teflon AF film mold and a UV-curable polyurethane acrylate prepolymer. ${ }^{[50]}$ We have utilized another fluoropolymer

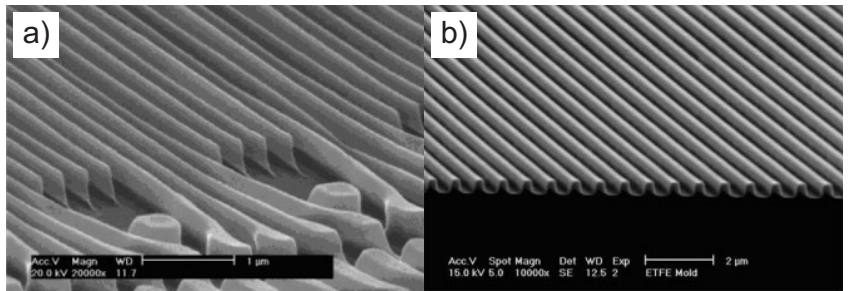

Figure 6. a) SEM image of $80 \mathrm{~nm}$ dense lines in a photocurable prepolymer solution molded by using a Teflon AF mold; scale bar: $1 \mu \mathrm{m}$. Reproduced with permission from [50]. Copyright 2004 American Chemical Society. b) SEM image of a $700 \mathrm{~nm}$ periodiocal grating structure in a UV-curable resist, imprinted by using a flexible sheet of a copolymer of ethylene and tetrafluoroethylene (ETFE) as a mold; scale bar: $2 \mu \mathrm{m}$ [52].

mold, ETFE or "Tefzel" (Dupont), which is a copolymer of ethylene and tetrafluoroethylene, that can be fabricated by imprinting using a hard template at a lower temperature of ca. $200^{\circ} \mathrm{C}$. Figure $6 \mathrm{~b}$ shows a grating pattern in a UV-curable epoxysilicone liquid resist ${ }^{[52]}$ imprinted by using the ETFE mold. A useful property of these fluoropolymers is that they can be used to make "copies" of the original mold, which may be difficult or expensive to fabricate. Recently, Choi et al. demonstrated a fluorinated organic-inorganic hybrid mold with thermal stability to over $350^{\circ} \mathrm{C}$ by using a nonhydrolytic sol-gel process. ${ }^{[33]}$

\section{NIL Resist}

Because imprint lithography makes a conformal replica of the surface relief patterns by mechanical embossing, the resist materials used in imprinting need to be easily deformable under an applied pressure and should have a sufficient mechanical strength as well as good mold-releasing properties to maintain their structural integrity during the de-molding process. For some applications, good etching properties are required for a subsequent RIE process.

Two material properties are important for the imprinting process. The resist material should have a Young's modulus lower than that of the mold during imprinting. The minimal pressure required to perform the imprint should be higher than the sheer modulus of the polymer. ${ }^{[54]}$ On the other hand, the low modulus of the resist material is necessary for it to be deformed by the mold. To complete the imprinting process within a practical time frame, the resist material should also have a sufficiently low viscosity. ${ }^{[55]}$ This requirement can be easily understood by considering the squeezed flow of a Newtonian liquid between plates that have a radius $R$ and a gap distance of $d{ }^{[56]}$ Such a model can approximate a process where a mold protrusion with size $R$ is imprinted into a resist of an initial thickness $d$ on a substrate. One can obtain a simple solution that expresses the pressure $(P)$ needed to obtain a certain imprinting speed $(\mathrm{d} h / \mathrm{d} t)$ in terms of these dimensions and the fluid viscosity $\eta: P=\left(3 \eta R^{2} / 4 d^{3}\right)(\mathrm{d} h / \mathrm{d} t)$. A numerical simulation based on the finite-difference method using the non-steady-state Navier-Stokes equation has also given such a relationship for simple periodic mold features. ${ }^{[57]}$ Integrating this equation can give the time required to halve the thickness of the fluidic layer (i.e., to imprint halfway through the feature height): $t=9 h R^{2} / 16 P d^{2}$. This simple analysis states that under a fixed pressure the imprinting time scales linearly with viscosity and quadratically with the pattern size. Therefore, the time required to imprint large-size patterns will be significantly longer than that required for nanoscale patterns.

\subsection{Thermal Plastic Resists}

For the plastic materials used in the thermal NIL process, the two requirements mentioned in the previous paragraph can be met simultaneously by raising the temperature above the glass transition temperature $\left(T_{\mathrm{g}}\right)$ of the polymer, so that both Young's modulus and the viscosity will drop by several orders of magnitude compared to their respective values at room temperature (Young's modulus for glassy polymers just below $T_{\mathrm{g}}$ is approximately constant over a wide range of polymers, at ca. $3 \times 10^{9} \mathrm{~Pa}$ ). In practice the temperature chosen for NIL is ca. $70-90^{\circ} \mathrm{C}$ above the $T_{\mathrm{g}}$, so that the polymer material reaches a viscous flow state ${ }^{[6]}$ In thermal NIL the mold and the imprinted polymer must be cooled to below the $T_{\mathrm{g}}$ of the polymer to preserve the imprinted pattern after the mold is removed. Because the $T_{\mathrm{g}}$ is the onset temperature for mo- 
lecular motion in polymers, factors that increase the energy required for molecular motion (e.g., intermolecular forces, intrachain steric hindrance such as branching or crosslinking, and bulky and stiff side groups) also increase the $T_{\mathrm{g}}$ value; those factors that decrease the energy requirement (e.g., flexible bonds, flexible side groups) lower the $T_{\mathrm{g}}$. These considerations can be exploited in choosing the desired $T_{\mathrm{g}}$ for the imprint resists. For many applications, it is desirable to use a lower temperature in processing. A reduction in temperature has to be compensated by corresponding increases in imprinting pressure and time to obtain satisfactory results. ${ }^{[58]}$

There is a trade-off between the imprinting temperature and the thermal stability, which is illustrated in the following example for polymers with different $T_{\mathrm{g}}$ values. Figure $7 \mathrm{a}$ shows patterns imprinted in poly(benzyl methacrylate) (molecular weight $\left(M_{\mathrm{w}}\right)$ ca. $\left.70000 \mathrm{~g} \mathrm{~mol}^{-1}, T_{\mathrm{g}}=54^{\circ} \mathrm{C}\right)$ at a temperature of $134{ }^{\circ} \mathrm{C}$, and Figure $7 \mathrm{~b}$ shows patterns imprinted in poly (cyclohexyl acrylate) $\left(M_{\mathrm{w}} \approx 150000 \mathrm{~g} \mathrm{~mol}^{-1}, T_{\mathrm{g}}=19^{\circ} \mathrm{C}\right)$ at $99^{\circ} \mathrm{C}$. In both cases an imprinting temperature of $T_{\mathrm{g}}+80^{\circ} \mathrm{C}$ was chosen, and the applied pressure was $5 \mathrm{MPa}$. Very good pattern definitions were obtained. Although a low- $T_{\mathrm{g}}$ material can be used in NIL for the sake of reducing the processing temperature, the imprinted patterns are also unstable and tend to deform at temperatures close to the imprinting temperature. Figure $7 \mathrm{c}$ shows pattern relaxation at room temperature, observed 10 days after the structure in Figure $7 \mathrm{~b}$ was imprinted (in this case the resist had a $T_{\mathrm{g}}$ of $19^{\circ} \mathrm{C}$, which is below room temperature). The patterned structures will relax faster at elevated temperatures, such as those encountered in a RIE chamber during subsequent removal of residual resist or pattern transfer into a substrate. Various alternatives have been exploited to lower the viscosity of thermoplastic materials, rendering them imprintable at temperatures close to room temperature. These include dissolving the polymer in its monomer ${ }^{[59]}$ or another solvent and using a PDMS stamp for solvent evaporation. ${ }^{[60]}$ From the example given above, it can be inferred that thermally curable or thermosetting polymers are excellent resist systems for NIL because of the possibility of low-pressure imprinting and good mechanical integrity after crosslinking by thermal treatment. An example of such a system is described in the following section.

The viscosity of a polymer material not only depends on the temperature, but also depends strongly on the polymer's $M_{\mathrm{w}}$
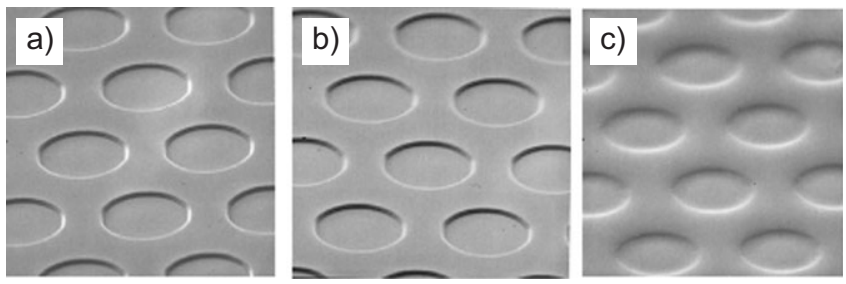

Figure 7. a,b) SEM images of patterns imprinted in a) poly(benzyl methacrylate) and b) poly(cyclohexyl acrylate). c) SEM image showing relaxation of poly(cyclohexyl acrylate) patterns 10 days after imprinting. Reproduced with permission from [6]. Copyright 2004 Institute of Physics. relative to the so-called critical molecular weight $\left(M_{\mathrm{c}}\right)$ of a given polymer. The $M_{\mathrm{c}}$ can be interpreted as the molecular weight at which a temporary network of entanglements spans over macroscopic dimensions. In practice, low-molecularweight polymers with $M_{\mathrm{w}}<M_{\mathrm{c}}$ can be imprinted at lower temperatures, lower pressures, or within shorter times. However, the absence of such a network of entanglements may lead to more brittle behavior, and could result in the fracture of the imprinted polymer features during the mold-separation step. Therefore, the choices of $T_{\mathrm{g}}$ and $M_{\mathrm{w}}$ are both important in maintaining the structural stability of the imprinted patterns. In addition, the stress build-up resulting from the applied pressure at a temperature below $T_{\mathrm{g}}$ after imprinting could strongly affect the integrity of the polymer pattern during mold separation. Hirai et al. have investigated this problem in detail. ${ }^{[61]}$ For more discussions on material rheology issues related to NIL, the readers are referred to Chapters 3 and 4 in an earlier publication. ${ }^{[56]}$

\subsection{UV-Curable Resists}

The high temperatures and pressures required for the nanoimprinting of thermoplastic materials could limit the throughput and application scope of the NIL technique. In addition, the thermal expansion mismatch between the mold and the substrate often presents an obstacle for pattern overlay over large substrates. An alternative is to use liquid precursors that can be cured by UV light at ambient temperatures. For such materials, the low Young modulus and low viscosity requirements are naturally satisfied. Because of the low viscosity of the monomer fluid the imprinting process is less sensitive to the effects of pattern density reported for NIL. Furthermore, the process allows the use of a small-area mold and the patterning of large-area substrates by a step-and-repeat process, similar to SFIL. The liquid resist developed for SFIL is a multicomponent solution containing a photoinitiator, a monomer with a high $\mathrm{Si}$ content to provide $\mathrm{O}_{2}$-RIE etch resistance, a difunctional monomer to allow crosslinking, and a low-molecular-weight monomer to reduce the viscosity of the solution. ${ }^{[62]}$ This resist formulation is referred to as an etch-barrier layer, which is the layer to be imprinted in SFIL. After patterning, this etch-barrier layer is used as a mask to transfer the pattern into a high-aspect-ratio pattern in an undercoating polymer resist layer. The organic monomer, $n$-butyl acrylate, serves as both a solvent and a mass-persistent component in the etchbarrier formulation. This assists in minimizing shrinkage during polymerization. The silylated monomer provides etch resistance in the $\mathrm{O}_{2}$ RIE process that transfers the low-aspectratio, high-resolution relief structures into high-aspect-ratio features in the undercoating resist layer. The crosslinker provides mechanical stability to the cured etch-barrier relief structure and also serves to improve the cohesive strength of the etch barrier, which are both necessary for a clean and reproducible separation. The resist formulation does not use a metal catalyst for curing, which makes it very attractive for 
integrated-circuit applications, where metal contaminations could affect or degrade the performance of semiconductor devices.

Two critical steps in NIL that follow the imprinting process are mold release and pattern transfer. After an ideal imprint, the large contact area between the mold and the resist gives rise to great adhesion forces. An ideal mold release involves resist shape integrity and complete mold-resist separation while the resist remains attached to the substrate. Apart from having good mold-releasing properties and a good mechanical integrity, an ideal resist should also have a good plasma-etching resistance for pattern transfer into substrate. Therefore, nanoimprint resist systems with combined mold-release and etch-resistance properties that allow fast and precise nanopatterning are highly desirable. Polymers developed specifically for NIL became commercially available in 2000. Currently, companies such as Micro Resist Technology (Germany), Molecular Imprint (Texas, USA), and Nanonex (New Jersey, USA) have developed resists for commercial use. Some resist materials are developed for specific techniques, such as the UV-curable liquid resist by Molecular Imprint for the SFIL process. Micro Resist Technology offers a few generic types of resists for NIL: the thermally curable mr-I 9000 works by free-radical polymerization of multifunctional aromatic allyl monomers; ${ }^{[63,64]}$ the UV-curable mr-L 6000 is essentially a chemically amplified negative-tone photoresist sensitive to near-UV exposure, comprising a multifunctional epoxidized novolak resin and a photoacid generator. Upon UV exposure the acids initiate the cationic polymerization of the epoxy resin, thereby forming a tightly crosslinked network. ${ }^{[64]}$

\subsection{New Material Developments}

The rapid development of NIL in recent years has also stimulated research towards new materials that are better suited for use as a nanoimprint resist. ${ }^{[65-67]}$ Three types of polymer systems that have recently been investigated in our group are described: a thermoplastic siloxane copolymer that provides excellent mold-releasing properties and the ability to produce high-resolution features with sufficient aspect ratios, a thermally curable PDMS-based liquid resist that can be imprinted and thermally crosslinked within $10 \mathrm{~s}$, and a UV-curable liquid resist based on cationic polymerization of silicone epoxies, which can be imprinted at low pressures using conventional contact aligners.

\subsubsection{Siloxane Copolymers}

The selection of a polymer system for use as NIL resist should consider critical aspects such as correct pattern replication, modest imprint temperature and pressure, proper mold release, and etch selectivity. The vast majority of thermal NIL methods uses homopolymer resists such as PMMA and polystyrene (PS), but these are susceptible to mold-sticking and fracture defects during mold release that are intolerable for many device applications. ${ }^{[68]}$ In this respect, the polymer should satisfy the seemingly contradictory requirements of having a low surface energy for easy de-molding whilst maintaining sufficient adhesion to the substrate. Although the mold surface is normally treated with low-surface-energy surfactants, ${ }^{[69]}$ the imprinted polymer still tends to adhere to the mold when imprinting high-density nanoscale structures or high aspect-ratio patterns. Materials that possess dual surface properties are needed to address these critical needs. Of special interest are the PDMS-organic block or graft copolymers. In contrast to PMMA, and organic polymers in general, siloxane copolymers exhibit significant differences by virtue of the highly open, flexible, and mobile $\mathrm{Si}-\mathrm{O}-\mathrm{Si}$ backbone. These include a low surface energy, low $T_{\mathrm{g}}$, and a high thermal stability. Furthermore, it is known that these copolymers undergo a microphase segregation above their $T_{\mathrm{g}} \mathrm{s}$ because of the unfavorable enthalpy of mixing. When cast or hot-pressed onto a high-surface-energy substrate such as silicon, glass, or metal the copolymer film forms an air/polymer interface enriched with lower-surface-energy component (PDMS block), and a polymer/substrate interface dominated by the higher-surfaceenergy component (organic block). ${ }^{[0]}$ The dual surface character makes these copolymers excellent candidate materials for NIL: they allow easy mold-resist separation and at the same time they exhibit good adhesion to the substrate. This duality is not possible with homopolymers. Siloxane copolymers offer another advantage over homopolymers, in that they have a strongly improved etching resistance because of the high Si content and the high strength of the Si-O bond. Our group has investigated a number of siloxane block and graft copolymers for NIL applications, including poly(dimethyl siloxane)-block-polystyrene (PDMS- $b$-PS), poly(dimethyl siloxane)-graft-poly(methacrylate)-co-poly(isobornyl acrylate), and poly(dimethyl siloxane)-graft-poly(methyl methacrylate) (PDMS-g-PMMA). ${ }^{[1]}$ Figure 8a shows a $250 \mathrm{~nm}$ linewidth grating imprinted in PDMS- $b$-PS. Figure $8 \mathrm{~b}$ and c shows a $70 \mathrm{~nm}$ linewidth grating mold made in a silicon nitride layer and the PDMS-g-PMIA resist imprinted at $170{ }^{\circ} \mathrm{C}$ and $1.3 \mathrm{MPa}$ pressure for $30 \mathrm{~s}$ with this mold. One of the tradeoffs for having a high silicon content is increased roughness as a result of phase separation. These siloxane copolymers all show excellent mold-releasing properties. To demonstrate the large-area performance of the copolymers, a full 4 inch $(1$ inch $=2.54 \mathrm{~cm})$ wafer with a $100 \mathrm{~nm}$ linewidth and spacing grating features on its entire surface was used for nanoimprinting. These large-area and dense features make it impossible to achieve mold-resist separation when using homopolymers (e.g, PMMA) without breaking the mold or the substrate because of the very strong adhesion forces, even with a fluorosilane-treated mold. With a PDMS- $g$-PMIA graft copolymer, excellent mold releasing was obtained. Figure 8d is a photograph showing strong light diffraction from the periodic gratings imprinted into the PDMS-g-PMIA when the sample was immersed in water (diffraction in air is in the UV range and invisible to the eye). In each of several successive imprints, there was no apparent gross delamination of the co- 

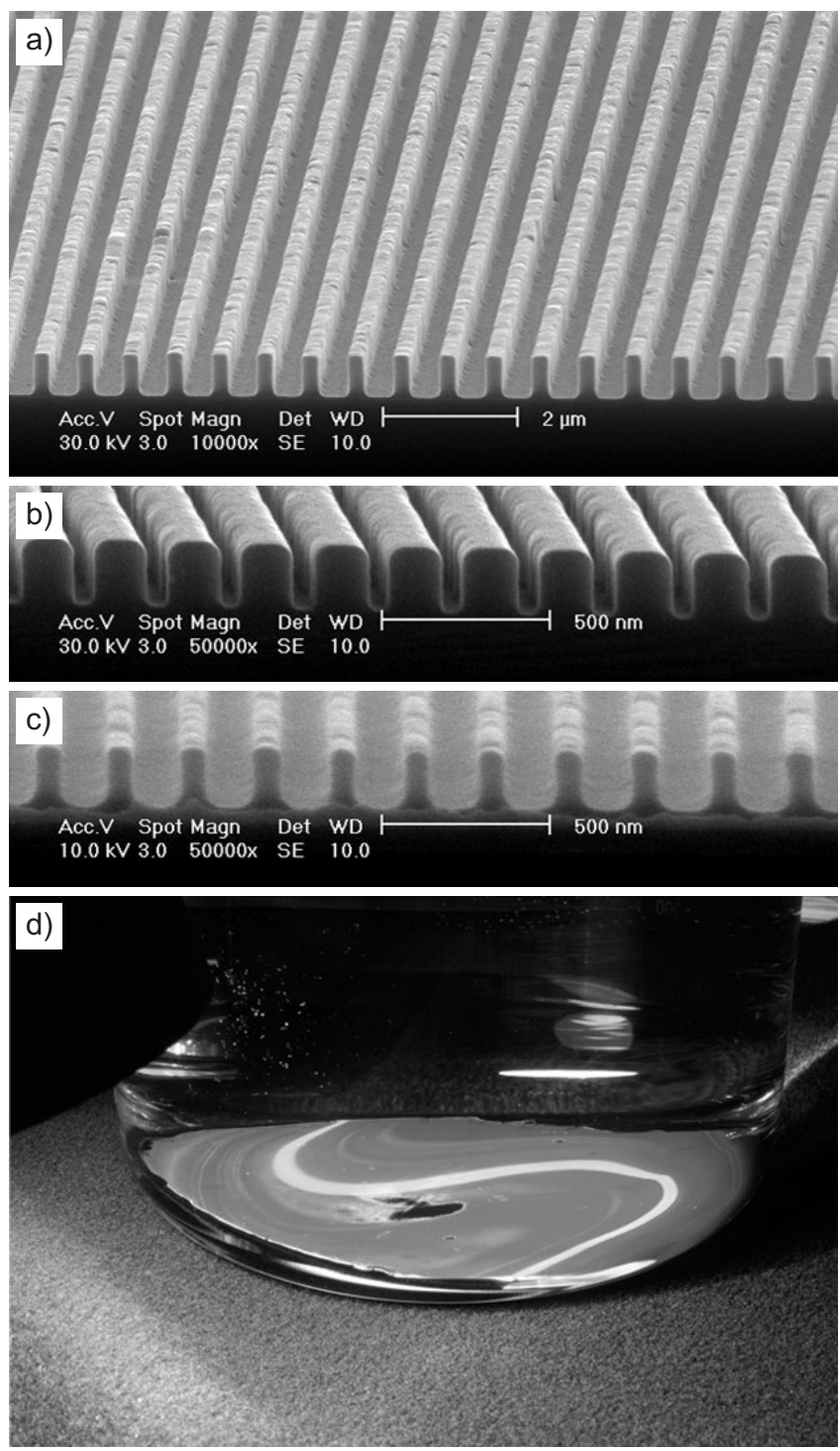

Figure 8. a-c) NIL results using a) a $250 \mathrm{~nm}$ linewidth PDMS- $b$-PS grating, b) a silicon nitride mold with $70 \mathrm{~nm}$ wide trenches, c) a $70 \mathrm{~nm}$ linewdith grating in PDMS-g-PMIA imprinted using the mold shown in (b). d) Strong light diffraction of an imprinted $200 \mathrm{~nm}$ period grating in a PDMS-g-PMIA copolymer on a 4 inch $(1 \mathrm{inch}=2.54 \mathrm{~cm})$ wafer immersed in water.

polymer, and as a result no need for solvent rinsing of the mold between imprints.

PDMS copolymers that have a sufficiently high silicone content provide a very good etching resistance for $\mathrm{O}_{2}$ RIE. This property can be exploited in a double-layer approach to create template structures suitable for the lift-off process, as shown in Figure 9a. Figure 9b and c shows the use of the bilayer resist approach to pattern on top of existing topography by using PDMS- $b$-PS and a PMMA undercoating layer. A $350 \mathrm{~nm}$ linewidth and spacing grating mold was used to imprint and lift-off metal lines. After the first lift-off was completed, the process was repeated in the orthogonal direction. The spin-coated PMMA underlayer served to planarize the nonflat substrate surface. Figure 9c shows the substrate after the deposition of a second metal layer, but before the lift-off process. The grid pattern of the combined metal layers is visible in the cross section, above which is the resist mask covered with metal. Figure 9d shows a large area of an etched Si hole array obtained by using the fabricated metal grid as a etch mask. Detailed studies of these copolymer systems as nanoimprint resists can be found elsewhere. ${ }^{[72]}$

\subsubsection{Fast Thermally Curable Liquid Resists}

The thermal plastic polymers used in NIL become viscous fluids when heated above their $T_{\mathrm{g}}$ values; however, the viscosity of heated polymers typically remains high, and thus the imprinting process requires significant pressures. As discussed previously these thermoplastic resists normally have a high tendency to stick to the mold, which seriously affects the fidelity and quality of the pattern definition. Furthermore, they do not offer the necessary etch resistance. Thermally curable monomers are an alternative to thermal plastics. These liquid materials can be imprinted in a short period of time at low pressures and temperatures. PDMS-type materials are a class of thermally curable materials widely used by many research groups, mainly in the context of soft lithography. In addition to its well-known transparency to UV and visible light along with its high biocompatibility, PDMS is a low-surface-energy (19.6 dyn $\mathrm{cm}^{-1}$ ) material, which allow an easy mold-release without causing any structural damage to the imprinted structures. Moreover, it possesses a very high resistance to oxygen plasma. However, its low Young modulus (ca. $2 \mathrm{MPa}$ for commercial Sylgard 184) impedes a good pattern definition at the nanoscale. This can be understood by considering the radius of curvature $(r)$ of a cured material with a certain modulus $(E)$ and surface energy $(\gamma)$, which can be estimated as $r=\gamma / \mathrm{E}^{\left[{ }^{[72]}\right.}$. It is easy to see that the low Young modulus of Sylgard PDMS makes it impossible to form nanopatterns with sharp corners. In addition, the low modulus can easily cause lateral collapse of the dense imprinted structures when patterns are smaller than $500 \mathrm{~nm}$. A higher-modulus (ca. $8 \mathrm{MPa}$ ) PDMS (h-PDMS) was developed by Schmith et al. to achieve the patterning of nanoscale structures for soft lithography. Unfortunately, the imprinting cycle (heating-cooling time) for this h-PDMS was too long (ca. $2 \mathrm{~h}$ ) for it to be used as an imprinting resist material. ${ }^{[73]}$ Following a similar strategy, Malaquin et al. also used a reformulated PDMS (prepolymer with a smaller chain length) as a thermal-curable resist for NIL. ${ }^{[74]}$ The shorter chain length of the prepolymer utilized in this formulation provided the stiffness necessary to replicate gratings with line widths as small as $200 \mathrm{~nm}$. However, the crosslinking time was about $60 \mathrm{~min}$ at $100{ }^{\circ} \mathrm{C}$ and $15 \mathrm{~min}$ at $150^{\circ} \mathrm{C}$ using a printing pressure of $100 \mathrm{MPa}$; and structures with an aspect ratio greater than 1 could not be obtained when replicating the $200 \mathrm{~nm}$ linewidth structures. ${ }^{[7]}$

A fast, thermal-curable liquid resist that can be imprinted under a low pressure with a high precision and throughput was recently developed in our group. ${ }^{[7]}$ This system is based 
a)

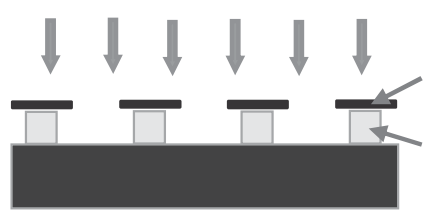

Deposit metal

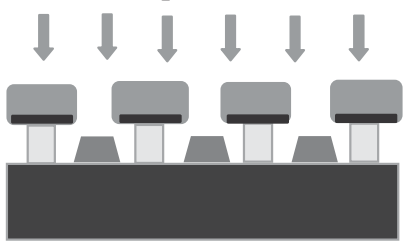

PS-PDMS

PMMA

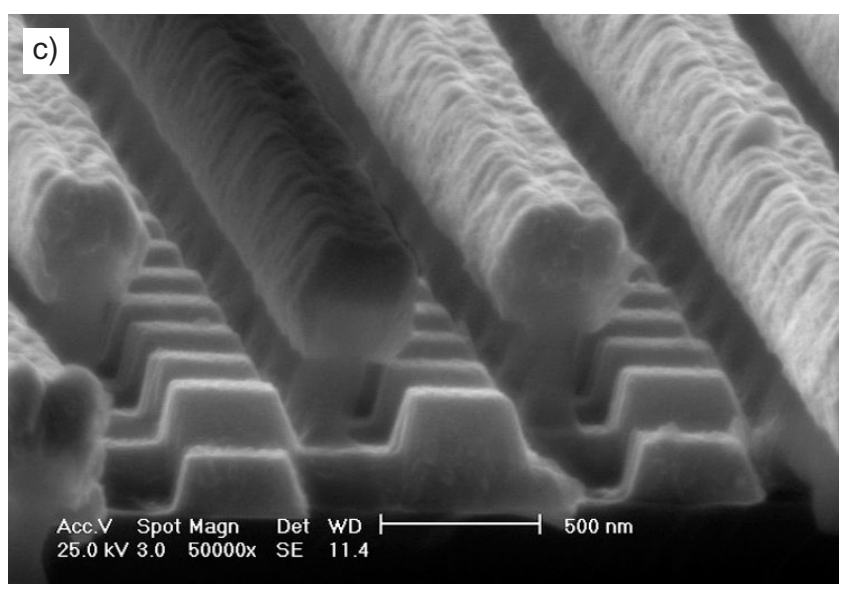

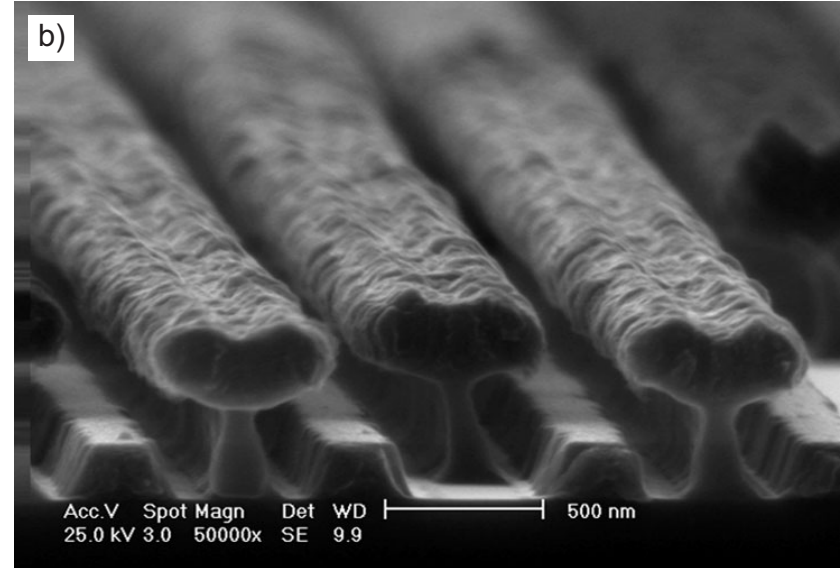

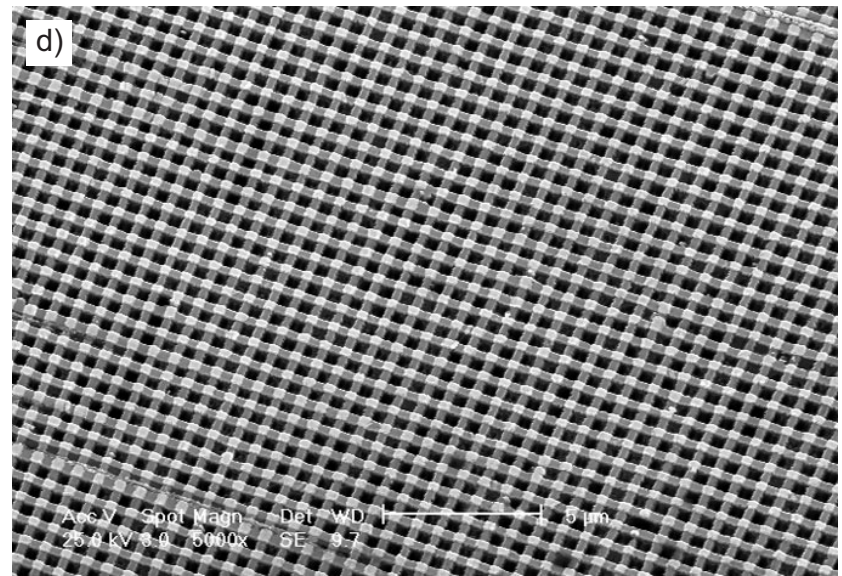

Figure 9. a) The $\mathrm{O}_{2}$ RIE resistance of copolymers, in this case PS-PDMS, allows for complex patterning by utilizing a planarization undercoating layer. Undercutting of the planarization layer eases the lift-off process. b) Metal deposition on the double-layer resist. c) Two-layer metal grid deposited by two complete NIL steps using a copolymer with a PMMA underlayer. d) Large-area etched Si hole array obtained by using the metal-grid as an etch mask; scale bar: $5 \mu \mathrm{m}$.

on the same hydrosilylation chemistry of siloxane polymers and consists of four basic chemical components: a vinyl-terminated PDMS polymer, a silyl-hydride-based $(\mathrm{Si}-\mathrm{H})$ dimethylsiloxane crosslinker, a platinum catalyst, and an inhibitor. The inhibitor is an unsaturated organic ester that keeps the catalyst inactive until the application of heat, which quickly deactivates the inhibitor and releases the catalyst in its active form. The high $\mathrm{Si}$ content in this polymer system guarantees that the resist has a high etching resistance in RIE processes. The liquid resist can be spin-coated onto a silicon wafer, forming a uniform thin film. The imprinting was done with moderate pressures in the range of $0.2-6.5 \mathrm{MPa}$ at room temperature, and crosslinking was performed at a temperature above $80^{\circ} \mathrm{C}$ within a minute. The short time required for curing the material is in sharp contrast with that of commercial PDMS, which requires curing for at least tens of minutes and sometimes hours. Also, by changing the molecular weight of the precursor and the percentage of silyl-hydride groups in the crosslinker, the Young modulus of the cured material can be tuned to values several times higher than that of commercial PDMS. The ability to tune the modulus imparts flexibility to the resist to- wards meeting different process conditions. With the increased modulus, structures with feature sizes ranging from several micrometers down to $70 \mathrm{~nm}$ could be achieved (Fig. 10). Also, by increasing the curing temperature to $120^{\circ} \mathrm{C}$, the material can be crosslinked within $10 \mathrm{~s}$ because of the rapid deactivation of the inhibitor. This is two orders of magnitude faster than previous thermally cured PMDS materials. The fast crosslinking time satisfies the need for high-speed patterning in practical applications. Importantly, the mold separation does not require cooling to room temperature, thereby further increasing the process throughput. Because of the low surface tension of the patterned film the mold separation is quite easy, even for high-aspect-ratio features. Details of these material properties can be found elsewhere. ${ }^{[76]}$ The crosslinked PDMS structures could also be used as molds for imprinting other resist materials or as stamps for microcontact printing, especially when sub-microscale features are desired. For example, earlier work $^{[76]}$ shows that the replicated PDMS sample can be used as a stamp to pattern the conductive polymer poly(3,4-ethylenedioxythiophene) (PEDOT) with sub-micrometer features by using a polymer inking and stamping technique. ${ }^{[19]}$ 

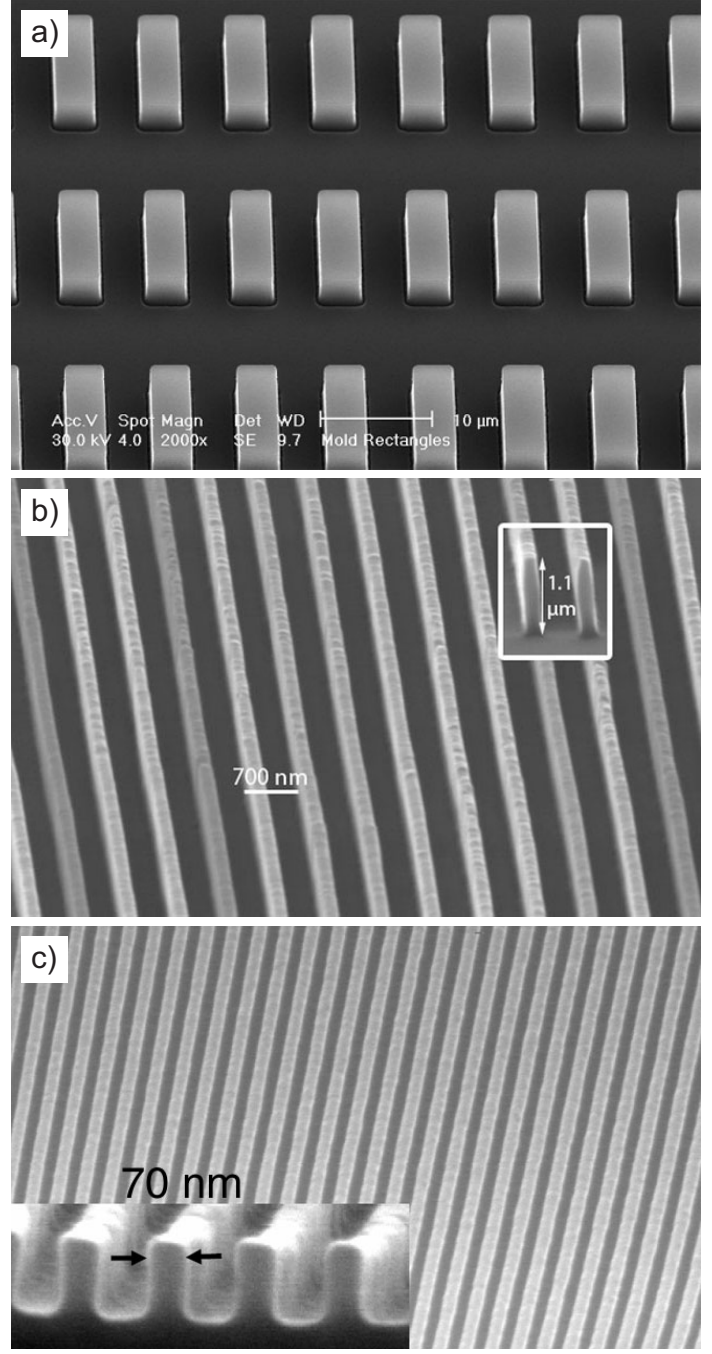

Figure 10. a-c) SEM images of imprinted PDMS-type thermally curable liquid resists, showing feature sizes ranging from (several) micrometers $(\mathrm{a}, \mathrm{b})$ down to $70 \mathrm{~nm}$ (c). Crosslinking by thermal curing can be achieved within $10 \mathrm{~s}$

\subsubsection{UV-Curable Liquid Resists for Room-Temperature Nanoimprinting}

For certain applications, materials systems that can be UVcured are preferred over thermally curable systems because the heating and cooling cycle of the latter can affect the throughput, and also have problematic issues that result from a thermal-expansion mismatch. The most widely used UV-curing formulations are based on free-radical polymerization of acrylic and methacrylic monomers because of their high reactivity, as used in SFIL. ${ }^{[62]}$ However, such materials systems suffer from oxygen sensitivity problems: dissolved oxygen scavenges free radical species, and thus inhibits the polymerization process at the resist surface at the onset of exposure. This prolongs the required exposure time. Oxygen diffusion in ambient condition causes a thin perimeter of undercured material surrounding the mold. Detailed kinetic studies have been carried out to evaluate the impact of oxygen on SFIL. ${ }^{[77,78]}$ Furthermore, the acrylate-based UV imprint resist has a large shrinkage upon curing (ca. 10\%), which may affect the pattern definition or resist adhesion on certain substrates, especially metals and plastics. On the other hand, as pointed out earlier this system does not require a metal catalyst, which makes it very attractive to Si-based microelectronics. An alternative formulation that uses vinyl ether has been investigated because it is based on cationic polymerization and less sensitive to oxygen. ${ }^{[79]}$ For the majority of other applications, the droplet dispensing method used to apply the liquid resist on a substrate in SFIL significantly limits the throughput of the nanopatterning process. The ability to spin-coat a uniform liquid resist onto a large-area substrate is highly desirable.

Our group has developed a UV-curable epoxysilicone material based on the cationic crosslinking of cycloaliphatic epoxies. ${ }^{[52]}$ This resist combines a number of desired features for nanoimprinting. Because cationic polymerization is not prone to oxygen inhibition, as compared to the free radical polymerization of acrylate monomers, fewer defects are expected. The resist exhibits a very good dry etching resistance because of the high silicon content. Furthermore, its very low shrinkage after curing (only a fraction of the acrylate system) allows reliable patterning. In addition, with a suitable undercoating polymer a very uniform liquid precursor can be formed simply by spin-coating, which also allows other processes, such as lift-off, to be easily performed.

The UV-curable liquid resist consists of a silicone-diepoxy monomer, a silicone cross-linking agent, and a photoacid generator. A typical liquid-resist formulation comprises diepoxy monomer $(94 \%, \mathrm{w} / \mathrm{w})$, crosslinkers $(5 \%)$, and a photoacid generator (PAG) (1\%). Organic solvents, such as propylene glycol monomethyl ether acetate (PGMEA), can be used to adjust the viscosity of the resist so that film thicknesses that range from one micrometer to $50 \mathrm{~nm}$ and below can be readily obtained. Although direct spin-coating of this resist onto a $\mathrm{Si}$ or oxide substrate causes dewetting of the thin liquid film, as one would expect for a very low viscosity liquid, we found that by using a suitable undercoating polymer layer (e.g., baked PMMA) or a photoresist (SU-8) to provide better matching of interfacial energies, stable and uniform liquid thin films can be formed on Si or any other substrate. This capability is critical to imprinting large-area (e.g., wafer-size) samples in a single step. With such a UV-curable nanoimprint resist, we were able to pattern both micro- and nanoscale structures at room temperature and at a pressure less than $0.1 \mathrm{MPa}$ using a conventional contact-exposure tool. Figure $11 \mathrm{~b}$ shows a recessed circular pattern with diameters of $20 \mu \mathrm{m}$ after imprinting and curing, and Figure 11c and d shows replicated nanoscale patterns, which demonstrates that patterns as small as $20 \mathrm{~nm}$ (limited by the feature resolution on the mold) can be easily achieved with a conventional contact aligner using this resist material. The UV-cured resist has very desirable plasma-etching characteristics, i.e., a very high resistance for $\mathrm{O}_{2}$ plasma etching, making it suitable for use as etch mask for pattern transferring into any underlying organic layers. 
a)
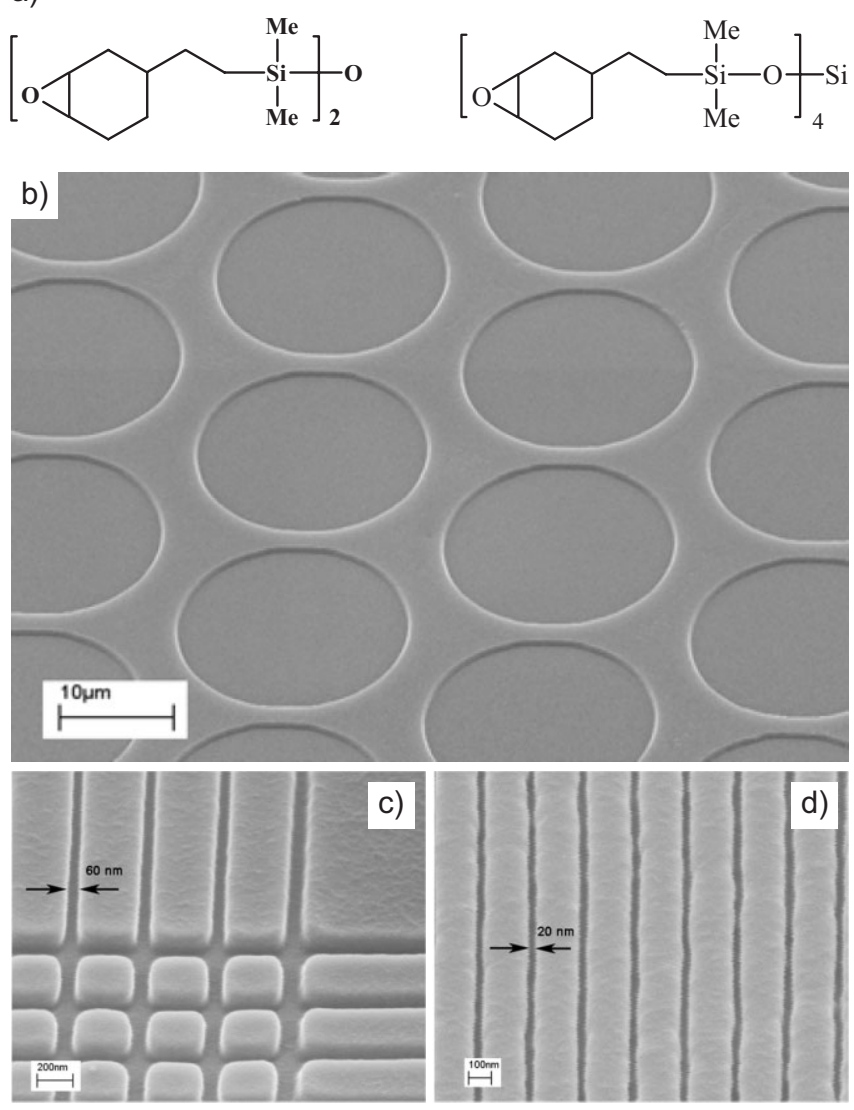

Figure 11. a) Structures of monomer and crosslink molecules. b-d) SEM images of imprinted and UV-cured resist patterns: b) $20 \mu \mathrm{m}$ diameter recessed patterns, c) sub-100 nm trench patterns, and d) $20 \mathrm{~nm}$ trenches [52].

\subsection{Other Imprintable Materials}

It should be recognized that NIL can not only be used to form patterns in a polymer resist, but can also be extended to create desired structures in many other polymer systems, especially those that have special functionalities, or can be used to form functional polymer device structures directly. Our group has imprinted 2D photonic-crystal nanostructures in nonlinear optical polymers. ${ }^{[25]}$ Pisignano et al. showed that conjugated polymers and oligomers can be imprinted at room temperature, and that the resulting nanostructures maintained active optical properties. ${ }^{[24,80]}$ The mechanism of room-temperature NIL was discussed by Lee and co-workers in an earlier article, which is based on the compression of free volume and plastic deformation in thermoplastic materials. ${ }^{[1]}$ Roomtemperature processes are highly desirable in patterning organic semiconductors to avoid oxygen incorporation and substitution into conjugated polymers, which occur at high temperature and can cause irreversible degradation of their optical and electrical properties. Li et al. patterned the conductive polymer PEDOT for use as electrodes in organic thinfilm transistors at high-resolutions. ${ }^{[19]}$ The process is based on a reverse-imprinting principle and is also carried at room temperature to preserve the conductive properties of the material. Recently, we have successfully imprinted a conjugatedpolymer-based electron donor layer to form a well-controlled nanoscale interface in an organic solar cell in order to improve charge separation and power conversion efficiency for organic photovoltaic applications. ${ }^{[82]}$

NIL also has many potential applications in microfluidics and biotechnology. Nielsen et al. demonstrated thermal NIL of the cyclic olefin copolymer "Topas", a thermoplastic material that is highly UV transparent and chemically resistant to hydrolysis, acids, and organic polar solvents, making it suitable for lab-on-a-chip applications. ${ }^{[83]}$ Cheng et al. imprinted the fluoropolymer "CYTOP", a low- $k$ dielectric material that also possesses interesting properties regarding the prevention of protein absorption. The authors utilized this property to pattern the motor proteins and used the imprinted CYTOP nanostructures as physical barriers to guide the motion of microtubules with an extremely high efficiency. ${ }^{[84]}$ Biodegradable polymers are attractive for many biomedical applications, such as DNA and protein analysis chips, as well as for supporting structures in tissue engineering. One popular example is poly-(L-lactic acid) (PLA) $\left(T_{\mathrm{g}} \approx 60^{\circ} \mathrm{C}\right)$. Hirai et al. demonstrated imprinting of commercial PLA plastics at $75^{\circ} \mathrm{C}$ to produce nanochannels and nanoscale hole arrays. ${ }^{[54]}$ This group has also showed imprinting of spin-on-glass for optical applications. Matsui et al. have demonstrated room-temperature NIL by using another type of spin-on-glass, hydrogen silsequioxane (HSQ), as the imprint resist material. ${ }^{[85,86]}$ The pressure needed for these inorganic materials was very high, because of HSQ's large Young modulus and high viscosity. On the other hand, its hydrophilic surface properties (similar to glass) and the ability to be nanopatterned at room temperature make it an ideal candidate material for creating microand nanofluidic applications. We developed a technique to form nanofluidic channels by direct imprinting of hydrophilic HSQ and sealing with another HSQ thin film, with all processing steps performed near room temperature (Fig. 12). The width of the channels is determined by the mold features, and the depth can be controlled through the imprinting pressure and time. ${ }^{[87]}$ The capability to produce nanoscale fluidic structures allows fundamental studies of ion or molecule transport in confined spaces. The study of nanofluidics could result in practical tools for the analysis of biomolecules, such as DNAs $^{[38,39]}$ and proteins.

\section{The Nanoimprint Process}

\subsection{The Cavity-Fill Process}

Here, an in-depth look at the imprinting step itself is taken, which is a critical step in the NIL process. To form the desired pattern, i.e., conforming to the nanoscale protrusion features on the mold, the resist material underneath the protrusions must be displaced and transported to nearby trenches or cav- 
a)

1. Spin coat $H S Q$ on substrate

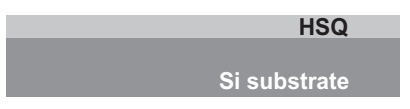

2. Direct imprinting of $H S Q$ film with a pressure of $1000 \mathrm{psi}$ at $25^{\circ} \mathrm{C}$.
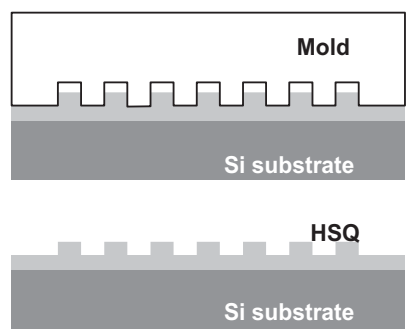

3. Transfer an HSQ film from an

$\mathrm{O}_{2}$ plasma treated PDMS stamp

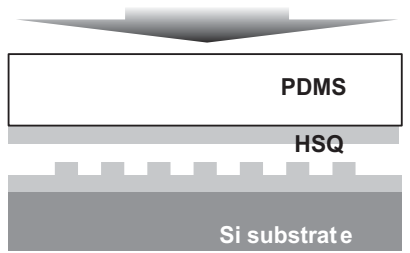

PDMS

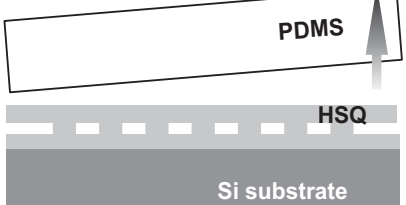

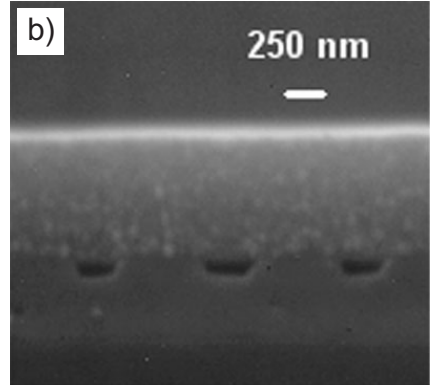

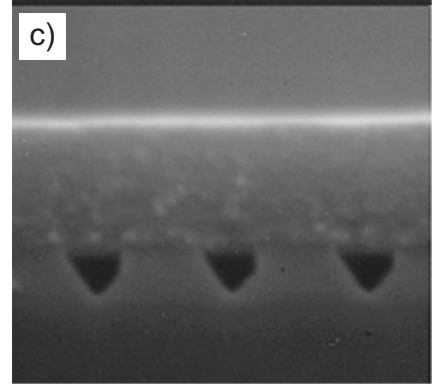

Figure 12. a) Schematic illustration of the fabrication of nanofluidic channels by direct imprinting of HSQ followed by bonding another HSQ layer. $b, c)$ SEM pictures of all-HSQ nanofluidic channels of different cross sections with a HSQ layer bond to the imprinted HSQ channels. Reproduced with permission from [87]. Copyright 2005 Transducer Research Foundation.

ities. A simple representation to depict the NIL process is to imagine pushing very sharp pins (with diameters down to $10 \mathrm{~nm}$ ) into a polymeric film. It is easy to understand that in NIL small-scale features are much easier to form than large ones, which is in sharp contrast with other lithographic techniques. Also, from the simple squeezed-flow model discussed earlier it can be seen that the time required for imprinting scales as a quadratic function of the pattern size, and therefore it takes significantly longer to imprint large-size patterns than nanopatterns. Therefore, the name "nanoimprint" is well-deserved, and accurately illustrates the process. The flow ability of the resist is perhaps the most important property in determining the imprinting conditions and the time required to complete the pattern replication.

Hirai et al. were the first to investigate the polymer deformation process by numerical simulations. ${ }^{[8]}$ The simulation results were compared with those obtained from NIL experiments, and the findings were summarized. ${ }^{\left[{ }^{89]}\right.}$ They have studied the pressures required for successful imprinting and the filling rate into the grooves of the mold as a function of the aspect ratio of the pattern, the initial thickness of the polymer, and the duty cycle of the structure for a periodic pattern. It was found that the required pressure increased not only for high-aspect-ratio patterns, but for low-aspect-ratio patterns as well. This is because for wide trenches the pressure is not evenly distributed as a result of the polymer flow resistance, and the polymers fill in from the edges with a slower filling rate than at the center of the cavity. The pressure also increases when the initial thickness of the polymer film decreases to less than twice the mold depth. This again can be attributed to the increased resistance of polymer flow in the confined nanofluidic channels formed between the mold pro- trusions and the substrate surface. These results agree very well with experimental observations. Based on these theoretical and experimental studies, Hirai's group has successfully demonstrated the imprinting of a high-aspect-ratio pattern having a $100 \mathrm{~nm}$ width and height of $860 \mathrm{~nm}$ by using a thick polymer resist layer (Fig. 13).

Several recent studies by Rowland et al. have investigated the impact of polymer material properties, mold geometry, and process conditions on polymer deformation, and further studied the impact of the polymer deformation mode on the replication time. ${ }^{[00-92]}$ They also found that when polymer flows vertically into an open cavity during imprinting, the polymer can deform as a single peak centered in the cavity, or as a dual peak, where each peak remains close to the vertical sidewalls, depending upon the geometry. The ratio of cavity

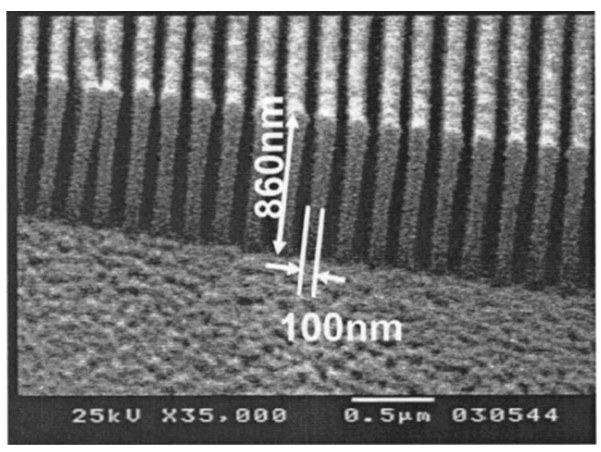

Figure 13. High-aspect-ratio grating pattern imprinted into a thick $(4.0 \mu \mathrm{m})$ PMMA-based polymer $\left(M_{\mathrm{w}}=100000 \mathrm{~g} \mathrm{~mol}^{-1}\right)$ on a Si substrate at $170^{\circ} \mathrm{C}$ and at a pressure of $20 \mathrm{MPa}$. Reproduced with permission from [89]. Copyright 2004 American Institute of Physics. 
width to film thickness modulates single versus dual peak cavity filling, regardless of the absolute size of the features and the pressure or temperature applied during imprinting. In general, when the ratio of cavity half width to initial polymer film thickness is less than unity, the polymer deforms as a single peak. For a ratio greater than unity, the polymer deforms as dual peaks. The cavity is filled fastest for a geometry at the single-peak/dual-peak threshold, with increasing fill times for conditions that deviate from this threshold, consistent with the findings of Hirai's group. Figure 14 shows simulation results for a polymer filling a cavity during thermal NIL for three different ratios of cavity size to polymer film thickness. ${ }^{[91]}$ For the leftmost and center case of Figure 14 , the flow is governed by shear near the mold corners. In both of these cases, there exists a plug of nondeforming polymer near the center of the cavity. In the case of the rightmost geometry, the polymer is squeezed beneath the mold, and this squeezed flow significantly limits the time required to fill this cavity. ${ }^{[92]}$

The ability to displace a viscous polymer and fill cavities can be demonstrated in another example, in which NIL is used to form an optical waveguide directly from a very thin polymer layer. The device is a polymer-based optical microring resonator, ${ }^{[68]}$ which can find applications in optical communication, label-free biosensing ${ }^{[93]}$ and high-frequency ultrasound detection. ${ }^{[94]}$ In this process an inverse pattern of the waveguide and the microring is fabricated as a mold, having a depth of ca. $2 \mu \mathrm{m}$ (Fig. 15a). For the NIL process, only a thin polymer layer with an initial thickness in the range of 200-300 $\mathrm{nm}$ is spin-coated, but the final waveguide thickness reaches ca. $2 \mu \mathrm{m}$ (Fig. 15b). This implies that the amount of polymer being displaced and transported to the mold trench is quite significant. Such a mode of imprinting is very different from that typically used in NIL, where nanoscale mold protrusions are imprinted into a polymer resist. Accordingly, the conditions for imprinting need to be modified by using a high-

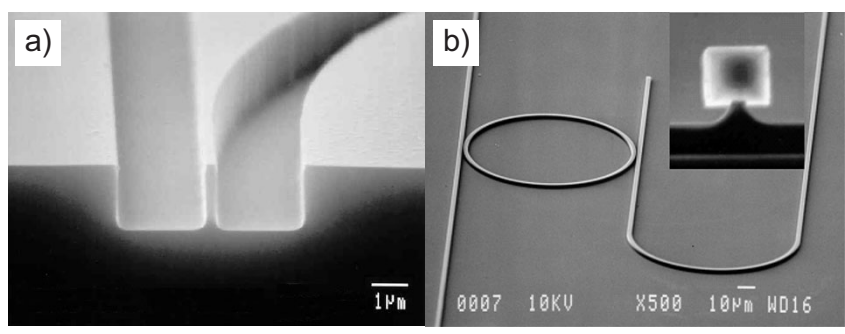

Figure 15. SEM images showing perspective view of a) a mold used to imprint the coupled polymer waveguides; scale bar: $1 \mu \mathrm{m}$ and b) an imprinted polymer microring resonator device; scale bar: $10 \mu \mathrm{m}$ (The insert shows a cross section of the waveguide after an undercutting wet etch).

er pressure and longer imprinting time to assist the polymer flow. ${ }^{[68]}$

Finally, Yu et al. developed a technique based on time-resolved scatterometry to perform in situ real-time monitoring of the cavity-filling process in NIL. ${ }^{[95]}$ In this approach, the authors used a surface relief grating structure as the imprinting mold, and monitored the diffracted light continuously as the mold penetrated into the polymer resist. The results matched the simulation very well, indicating that this technique is a powerful tool for in situ NIL process control.

\section{Variations of NIL Processess}

In recent years various new approaches aimed at enhancing the capability of NIL have been studied. Roller NIL was proposed to provide better uniformity and decrease the printing force, and demonstrated sub-100 nm feature transfer by using a hard mold. ${ }^{[96]}$ The rigiflex mold was developed to provide a better conformal contact with the substrate and to reduce the pressure required during imprint. ${ }^{[43]}$ This approach can also be extended for roll-to-roll imprinting of nanostructures. In

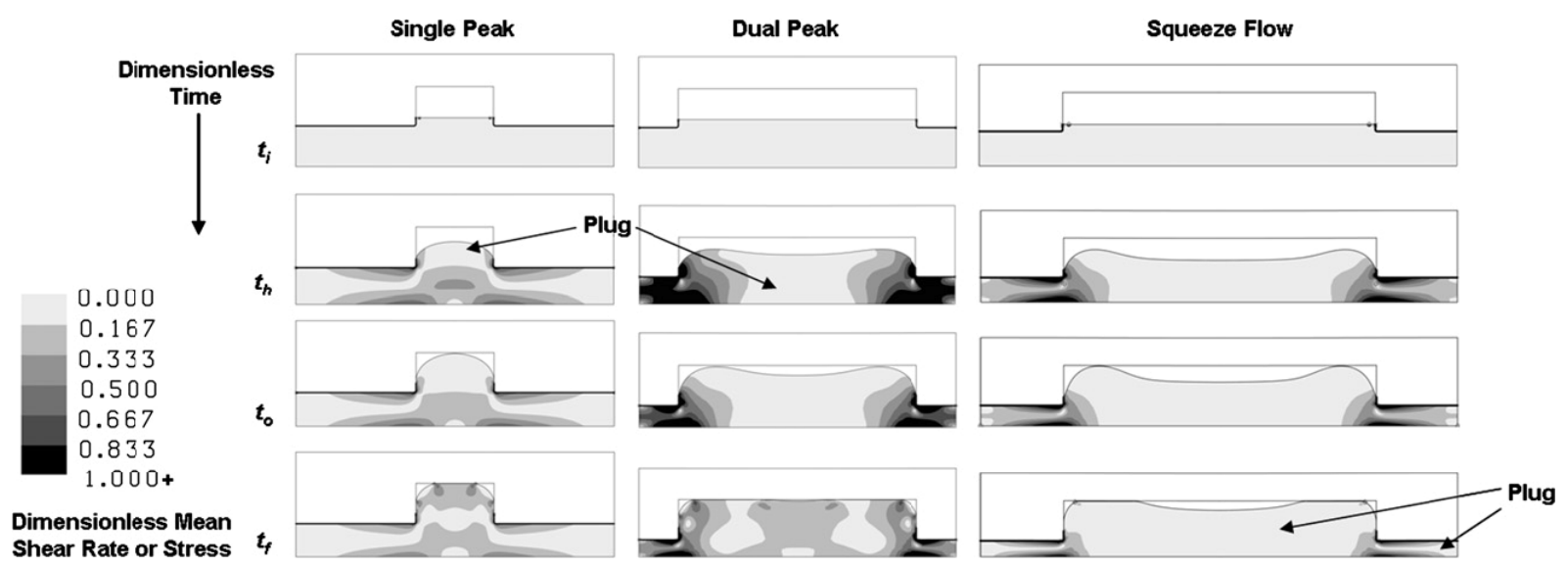

Figure 14. Simulations showing the temporal evolution of polymer flow into an imprint mold cavity for three different ratios of polymer cavity size to polymer thickness. In general, the ratio of cavity half width to polymer film thickness determines whether a single peak or dual peak will occur for the polymer deformation. The polymer gray scale indicates shear: a darker color represents higher shear. The location of polymer shear modulates the polymer deformation mode, which in turn modulates the cavity-filling time. Reproduced with permission from [90]. Copyright 2004 Institute of Physics. 
SFIL, a reverse-tone bilayer approach was developed to enhance the reliability in fabricating high-aspect-ratio structures. ${ }^{[97]}$

\subsection{Reverse-Nanoimprinting}

Borzenko et al. developed a polymer bonding technique to reduce the temperature and pressure used in NIL and to reduce the impact of limited polymer transport on pattern forma-

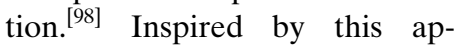

proach, our group developed a reverse-nanoimprint technique to address the issues of patterning on topographies and on a flexible substrate. Reverse nanoimprinting is based on the following consideration: when a polymer film is spin-coated onto a mold, the polymer will fill up the trench regions of the surface relief patterns. This means that a replica of the mold pattern is formed in the polymer film simply by spin-coating. If this film can then be transferred from the mold to a substrate, patterned structures are obtained (Fig. 16a). Figure 16b shows a $350 \mathrm{~nm}$ linespacing PMMA grating obtained by the reverse imprint process at a temperature of $105^{\circ} \mathrm{C}$, i.e., at the $T_{\mathrm{g}}$ of PMMA. The key to successful film transfer lies in the fact that the mold has a lower surface energy than the substrate, so that the polymer film has a better adhesion to the substrate and can therefore be detached from the mold.

Because coating of the polymer resist on the substrate is not required in reverse nanoimprinting, it is possible to use this technique to transfer patterns onto substrates that are not suitable for spin-coating or have surface topographies (Fig. 17). Our group has demonstrated that it can be applied to flexible substrates and substrates that have prefabricated structures (e.g., nonflat or prepatterned surfaces) to create nanoscale patterns. ${ }^{[99]}$ Figure 18 a shows a reverse-imprinted polycarbonate grating structure that is suspended over etched
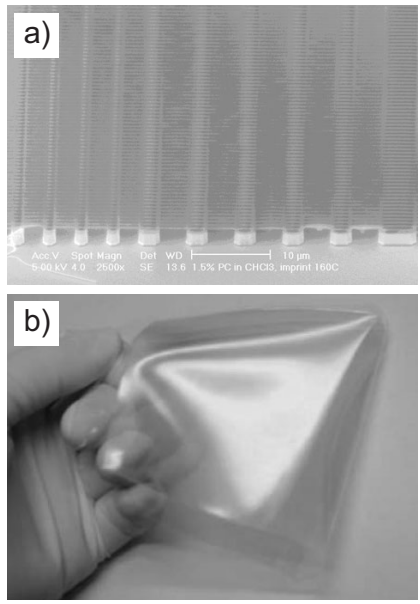

Figure 18. a) SEM image of $700 \mathrm{~nm}$ period grating in polycarbonate reverse-imprinted on topographies; scale bar: $10 \mu \mathrm{m}$. b) Period grating $(700 \mathrm{~nm})$ in PMMA over a $10 \mathrm{~cm}$ area, reverse imprinted onto a flexible PVC thin film, showing strong and uniform light diffraction across the whole printing area.

channels on a Si substrate, and Figure 18b shows a large-area (10 cm size) PMMA grating reverse-imprinted over a poly(vinyl chloride) (PVC) thin film. The results showed high yields and little defects. Bright rainbow colors can be observed from the flexible

a)
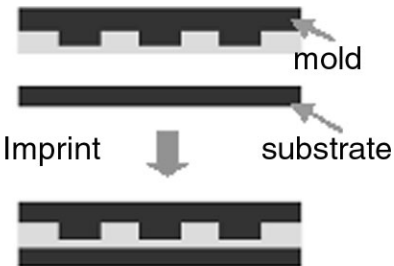

Separate

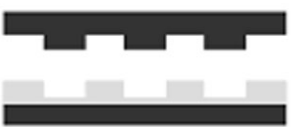
Figure 16. a) Schematic illustration of the reverse nanoimprint process. b) SEM picture of reverse-
imprinted PMMA gratings with a $350 \mathrm{~nm}$ linewidth/spacing.

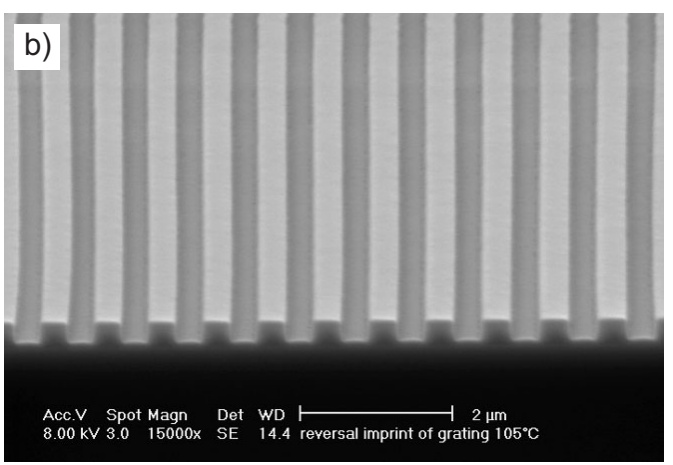

film as a result of the strong light diffraction from the imprinted grating structures on its surface. The reverseimprinting technique also offers a simple method to fabricate $3 \mathrm{D}$ polymer nanostructures by simply repeating the process to build up the structure in a layer-by-layer fashion. ${ }^{[99,100]}$ By using two molds, Kong et al. showed another way of constructing simple 3D structures using the reverse-imprinting principle. $^{[101]}$

The reverse-nanoimprinting method can be operated in another mode when 
the depth of the mold feature is large compared to the coated polymer film. In this case, the coated polymer can not planarize the relief features on the mold surface, and when pressed against a substrate only the protrusion features on top of the mold are transferred to the substrate material. ${ }^{[102,103]}$ This is similar to the inking process, but the stamp has nanoscale surface relief features. The polymer inking method is essentially an additive patterning technique that does not require further processing steps. This feature is very attractive for patterning polymers with special functionalities that are sensitive to chemical or plasma processing. Such examples include conductive polymers and conjugated organic materials. Recently, the conductive polymer PEDOT has been patterned by the polymer inking technique to function as electrodes for organic electronic applications. ${ }^{[19]}$ Lee et al. demonstrated the patterning of a bilayer of metal and polymer. ${ }^{[104]}$

\subsection{Combined Nanoimprint-and-Photolithography}

NIL works effectively for nanoscale features, but experiences difficulties when replicating larger features. On the other hand, a general lithographic technique should be capable of producing both large and small features in various combinations and with various pattern densities. Previous studies have shown that defects or even pattern failures in the form of incomplete pattern transfer can occur as a result of the high viscosity of the polymer melt and the varied pattern densities on the mold. ${ }^{[105]}$ A mix-and-match lithography approach that uses two separate lithography steps has been proposed to address this problem, ${ }^{[106]}$ at the expense of an increased complexity and number of processing steps as well as the requirement of pattern registration between the two separate steps. Our group developed a technique to solve this problem by introducing a hybrid mask concept and combining NIL with photolithography (CNP). ${ }^{[107]}$ As shown in Figure 19a, the hybrid mold is made of a UV-transparent material and acts as both an NIL mold and as a photolithography mask. Protrusions are made on the mold for imprinting nanoscale features, while metal pads are embedded into the mold and serve as a photolithography metal mask to replicate the large patterns. Detailed fabrication procedures have been reported elsewhere. ${ }^{[108]}$ In the CNP process, the hybrid mold is first imprinted into the resist layer by pressure, and subsequently the entire mold-substrate assembly is exposed to UV radiation. After the hybrid mold and the substrate are separated, the substrate is immersed in a developer solution to remove unexposed resist (i.e., resist that was blocked by the metal pads). After development, both large and nanoscale patterns are created in the polymer resist in one step. The authors have demonstrated the effectiveness of this technique by using a negative-tone photoresist, and have also fabricated nanoelectrode structures with drastically different length scales, from $150 \mu \mathrm{m}$ down to tens of nanometers (Fig. 19b). Such structures were used in making nanoscale organic thin-film transistors. ${ }^{[108]}$ The CNP process offers several advantages: First, the nanoscale protrusion features on the hybrid mold only need to displace a very small amount of polymer, which ensures a low-pressure process. Second, by forming the large patterns as a photomask (i.e., making them as metal pads), the residue layer thickness distribution is simplified, which can ease the residue removal step significantly. Third, as a further improvement, if the metal mask used for etching the mold is left on top of the mold protrusions it can prevent the exposure of the resist layer underneath. The unexposed residual layer can be removed in a developer solution without additional $\mathrm{O}_{2}$ dry etching steps. ${ }^{[107]}$ In this manner it is possible to eliminate the separate residual removal step in NIL completely, and simultaneously the nonuniform residual layer thickness problem could be solved altogether. In addition, the metal layer on the hybrid mold used in CNP serves as better registration marks, as compared with the relief structures used in SFIL or NIL. Because the latter can not create a sufficient refractive-index contrast upon intimate contact with a polymer layer that has an index close to the silica or quartz mold, registration between different lithography layers is difficult. To take advantage of the high-contrast alignment marks in CNP, one can design grating patterns on the mold and on substrate and use the Moiré interference technique to achieve a high-accuracy alignment.

\section{Conclusion}

The nanoimprint technique has enabled parallel nanoscale patterning with ultrahigh resolutions. The simplicity of this method has made it appealing to researchers in various fields. Hopefully, this Review will provide the readers with an overview of some aspects of the rapid developments in the field of nanoimprint technology in recent years, especially where new materials are concerned, but it is by no means meant to be exhaustive. Novel techniques based on the concept of mechanical printing or embossing are appearing rapidly. New variations of the technique, aimed at different applications, have also been developed at a fast pace. It is certain that advances in (new) imprinting materials will fuel further developments in this field. One exciting opportunity is the development of truly continuous roll-to-roll imprinting of nanostructures, which could provide unprecedented throughput for many practical applications. Hopefully, more researchers will be inspired to explore these techniques, and to exploit many new possibilities in the future.

Received: April 24, 2006

Revised: August 8, 2006 Published online: January 25, 2007 
a)
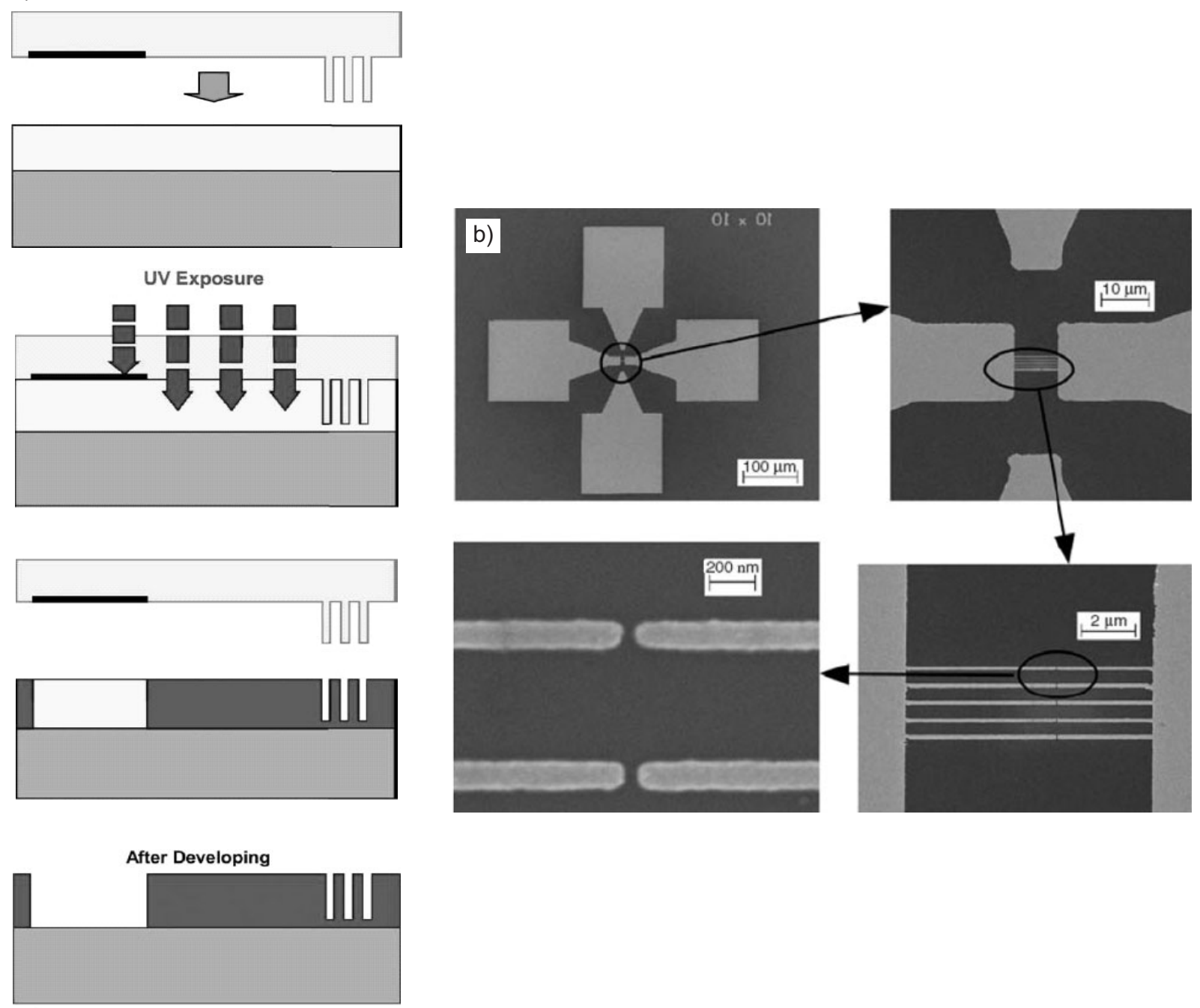

Figure 19. a) Schematic illustration of the CNP technique, using a hybrid mask mold for one-step lithography of both large and nanopatterns. Reproduced with permission from [107]. Copyright 2004 Elsevier. b) SEM images of a finger-shaped nanoelectrode with large metal pads patterned by the CNP approach. Reproduced with permission from [108]. Copyright 2006 Institute of Physics.

[1] Y. Xia, G. M. Whitesides, Polym. Mater. Sci. Eng. 1997, 77, 596.

[2] S. Y. Chou, P. R. Krauss, P. J. Renstrom, Appl. Phys. Lett. 1995, 67, 3114.

[3] S. C. Minne, P. Flueckiger, H. T. Soh, C. F. Quate, J. Vac. Sci. Technol. B 1995, 13, 1380.

[4] R. D. Piner, J. Zhu, F. Xu, S. Hong, C. A. Mirkin, Science 1999, 283, 661.

[5] B. D. Gates, Q. B. Xu, M. Stewart, D. Ryan, C. G. Willson, G. M. Whitesides, Chem. Rev. 2005, 105, 1171.

[6] L. J. Guo, J. Phys. D 2004, 37, R123.

[7] S. Y. Chou, P. R. Krauss, P. J. Renstrom, Science 1996, 272, 85.

[8] S. Y. Chou, P. R. Krauss, W. Zhang, L. J. Guo, L. Zhuang, J. Vac. Sci. Technol. B 1997, 15, 2897.

[9] P. Ruchhoeft, M. Colburn, B. Choi, H. Nounu, S. Johnson, T. Bailey, S. Damle, M. Stewart, J. Ekerdt, S. V. Sreenivasan, J. C. Wolfe, C. G. Willson, J. Vac. Sci. Technol. B 1999, 17, 2965.
[10] M. D. Stewart, S. C. Johnson, S. V. Sreenivasan, D. J. Resnick, C. G. Willson, J. Microlithogr., Microfabr., Microsyst. 2005, 4, 011002.

[11] D. J. Resnick, W. J. Dauksher, D. Mancini, K. J. Nordquist, T. C. Bailey, S. Johnson, N. Stacey, J. G. Ekerdt, C. G. Willson, S. V. Sreenivasan, N. Schumaker, J. Vac. Sci. Technol. B 2003, 21, 2624.

[12] Special Report, Technol. Rev. 2003, 106, 36.

[13] T. C. Bailey, D. J. Resnick, D. Mancini, K. J. Nordquist, W. J. Dauksher, E. Ainley, A. Talin, K. Gehoski, J. H. Baker, B. J. Choi, S. Johnson, M. Colburn, M. Meissl, S. V. Sreenivasan, J. G. Ekerdt, C. G. Willson, Microelectron. Eng. 2002, 61-2, 461.

[14] W. J. Dauksher, K. J. Nordquist, D. P. Mancini, D. J. Resnick, J. H. Baker, A. E. Hooper, A. A. Talin, T. C. Bailey, A. M. Lemonds, S. V. Sreenivasan, J. G. Ekerdt, C. G. Willson, J. Vac. Sci. Technol. B 2002, 20, 2857.

[15] T. Bailey, B. J. Choi, M. Colburn, M. Meissl, S. Shaya, J. G. Ekerdt, S. V. Sreenivasan, C. G. Willson, J. Vac. Sci. Technol. B 2000, 18, 3572. 
[16] A. Fuchs, B. Vratzov, T. Wahlbrink, Y. Georgiev, H. Kurz, J. Vac. Sci. Technol. B 2004, 22, 3242.

[17] M. C. McAlpine, R. S. Friedman, D. M. Lieber, Nano Lett. 2003, 3 , 443.

[18] C. C. Cedeno, J. Seekamp, A. P. Kam, T. Hoffmann, S. Zankovych, C. M. S. Torres, C. Menozzi, M. Cavallini, M. Murgia, G. Ruani, F. Biscarini, M. Behl, R. Zentel, J. Ahopelto, Microelectron. Eng. 2002, 61-2, 25

[19] D. Li, L. J. Guo, Appl. Phys. Lett. 2006, 88, 63513

[20] L. J. Guo, P. R. Krauss, S. Y. Chou, Appl. Phys. Lett. 1997, 71, 1881.

[21] W. Zhang, S. Y. Chou, Appl. Phys. Lett. 2003, 83, 1632.

[22] D. S. Macintyre, Y. Chen, D. Gourlay, E. Boyd, D. Moran, X. Cao, K. Elgaid, C. R. Stanley, I. Thayne, S. Thoms, J. Vac. Sci. Technol. B 2003, 21, 2783.

[23] D. Pisignano, L. Persano, E. Mele, P. Visconti, M. Anni, G. Gigli, R. Cingolani, L. Favaretto, G. Barbarella, Synth. Met. 2005, 153, 237.

[24] D. Pisignano, L. Persano, M. F. Raganato, P. Visconti, R. Cingolani, G. Barbarella, L. Favaretto, G. Gigli, Adv. Mater. 2004, 16, 525.

[25] L. J. Guo, X. Cheng, C. Y. Chao, J. Mod. Opt. 2002, 49, 663.

[26] X. Cheng, Y. T. Hong, J. Kanicki, L. J. Guo, J. Vac. Sci. Technol. B 2002, 20, 2877.

[27] P. C. Kao, S. Y. Chu, T. Y. Chen, C. Y. Zhan, F. C. Hong, C. Y. Chang, L. C. Hsu, W. C. Liao, M. H. Hon, IEEE Trans. Electron Devices 2005, 52, 1722 .

[28] J. Wang, H. Kostal, Laser Focus World 2005, 41, 76.

[29] J. J. Wang, W. Zhang, X. G. Deng, J. D. Deng, F. Liu, P. Sciortino, L. Chen, Opt. Lett. 2005, 30, 195.

[30] S. W. Ahn, K. D. Lee, J. S. Kim, S. H. Kim, J. D. Park, S. H. Lee, P. W. Yoon, Nanotechnology 2005, 16, 1874.

[31] Y. Ekinci, H. H. Solak, C. David, H. Sigg, Opt. Express 2006, 14, 2323.

[32] S. Y. Chou, Proc. IEEE 1997, 85, 652

[33] W. Wu, B. Cui, X. Y. Sun, W. Zhang, L. Zhuang, L. S. Kong, S. Y. Chou, J. Vac. Sci. Technol. B 1998, 16, 3825.

[34] J. I. Martin, J. Nogues, K. Liu, J. L. Vicent, I. K. Schuller, J. Magn. Magn. Mater. 2003, 256, 449.

[35] T. Glinsner, P. Hangweier, H. Luesebrink, P. Dorsey, A. Homola, D. Wachenschwanz, Solid State Technol. 2005, 48, 51 .

[36] G. M. McClelland, M. W. Hart, C. T. Rettner, M. E. Best, K. R. Carter, B. D. Terris, Appl. Phys. Lett. 2002, 81, 1483.

[37] Z. J. Hu, G. Baralia, V. Bayot, J. F. Gohy, A. M. Jonas, Nano Lett. 2005, 5,1738 .

[38] H. Cao, Z. N. Yu, J. Wang, J. O. Tegenfeldt, R. H. Austin, E. Chen, W. Wu, S. Y. Chou, Appl. Phys. Lett. 2002, 81,174

[39] L. J. Guo, X. Cheng, C. F. Chou, Nano Lett. 2004, 4, 69.

[40] J. D. Hoff, L. J. Cheng, E. Meyhofer, L. J. Guo, A. J. Hunt, Nano Lett. 2004, 4, 853.

[41] D. Falconnet, D. Pasqui, S. Park, R. Eckert, H. Schift, J. Gobrecht, R. Barbucci, M. Textor, Nano Lett. 2004, 4, 1909.

[42] W. Hu, E. K. F. Yim, R. M. Reano, K. W. Leong, S. W. Pang, J. Vac. Sci. Technol. B 2005, 23, 2984.

[43] D. Suh, S.-J. Choi, H. H. Lee, Adv. Mater. 2005, 17, 1554

[44] D. Y. Khang, H. Yoon, H. H. Lee, Adv. Mater. 2001, 13, 749.

[45] B. D. Lucas, J. Kim, L. J. Guo, unpublished.

[46] M. D. Austin, H. X. Ge, W. Wu, M. T. Li, Z. N. Yu, D. Wasserman, S. A. Lyon, S. Y. Chou, Appl. Phys. Lett. 2004, 84, 5299.

[47] M. Tormen, A. Carpentiero, L. Vaccari, M. Altissimo, E. Ferrari, D. Cojoc, E. Di Fabrizio, J. Vac. Sci. Technol. B 2005, 23, 2920.

[48] G. Y. Jung, Z. Y. Li, W. Wu, Y. Chen, D. L. Olynick, S. Y. Wang, W. M. Tong, R. S. Williams, Langmuir 2005, 21, 1158.

[49] H. Schift, S. Saxer, S. Park, C. Padeste, U. Pieles, J. Gobrecht, Nanotechnology 2005, 16, S171.

[50] D. Y. Khang, H. H. Lee, Langmuir 2004, 20, 2445.

[51] D. Y. Khang, H. Kang, T. Kim, H. H. Lee, Nano Lett. 2004, 4, 633.

[52] X. Cheng, L. J. Guo, P. F. Fu, Adv. Mater. 2005, 17, 1419.
[53] D. G. Choi, J. H. Jeong, Y. S. Sim, E. S. Lee, W. S. Kim, B. S. Bae, Langmuir 2005, 21, 9390.

[54] Y. Hirai, Y. Tanaka, J. Photopolym. Sci. Technol. 2002, 15, 475.

[55] Y. Hirai, J. Photopolym. Sci. Technol. 2005, 18, 551.

[56] Alternative Lithography: Unleashing the Potentials of Nanotechnology (Ed: C. M. Sotomayor-Torres), Kluwer Academic/Plenum, New York 2003, pp. 36-41.

[57] L. Wu, S. Y. Chou, in Technical Digest of 47th EIPBN Conf. 2003, Tampa, FL 2003, 293.

[58] H. C. Scheer, N. Bogdanski, M. Wissen, T. Konishi, Y. Hirai, J. Vac. Sci. Technol. B 2005, 23, 2963.

[59] G. Y. Jung, S. Ganapathiappan, X. Li, D. A. A. Ohlberg, D. L. Olynick, Y. Chen, W. M. Tong, R. S. Williams, Appl. Phys. A 2004, 78, 1169.

[60] D. Y. Khang, H. H. Lee, Appl. Phys. Lett. 2000, 76, 870.

[61] Y. Hirai, S. Yoshida, N. Takagi, J. Vac. Sci. Technol. B 2003, 21, 2765.

[62] M. Colburn, S. Johnson, M. Stewart, S. Damle, T. Bailey, B. Choi, M. Wedlake, T. Michaelson, S. Sreenivasan, J. Ekerdt, Proc. SPIEInt. Soc. Opt. Eng. 1999, 3676, 379.

[63] H. Schulz, D. Lyebyedyev, H. C. Scheer, K. Pfeiffer, G. Bleidiessel, G. Grutzner, J. Ahopelto, J. Vac. Sci. Technol. B 2000, 18, 3582.

[64] K. Pfeiffer, F. Reuther, M. Fink, G. Gruetzner, P. Carlberg, I. Maximov, L. Montelius, J. Seekamp, S. Zankovych, C. M. Sotomayor-Torres, H. Schulz, H. C. Scheer, Microelectron. Eng. 2003, 67-68, 266.

[65] M. D. Stewart, C. G. Willson, MRS Bull. 2005, 30, 947

[66] F. Reuther, J. Photopolym. Sci. Technol. 2005, 18, 525.

[67] N. Sakai, J. Taniguchi, K. Kawaguchi, M. Ohtaguchi, T. Hirasawa, J. Photopolym. Sci. Technol. 2005, 18, 531.

[68] C. Y. Chao, L. J. Guo, J. Vac. Sci. Technol. B 2002, 20, 2862.

[69] M. Beck, M. Graczyk, I. Maximov, E. L. Sarwe, T. G. I. Ling, M. Keil, L. Montelius, Microelectron. Eng. 2002, 61-2, 441.

[70] P. Fu, M. K. Tomalia, Macromolecules 2004, 37, 267.

[71] P. Choi, P. Fu, L. J. Guo, Adv. Funct. Mater., doi: 10.1002/ adfm.200600257.

[72] C. Y. Hui, A. Jagota, Y. Y. Lin, E. J. Kramer, Langmuir 2002, 18, 1394.

[73] H. Schmid, B. Michel, Macromolecules 2000, 33, 3042.

[74] L. Malaquin, F. Carcenac, C. Vieu, M. Mauzac, Microelectron. Eng. 2002, 61-2, 379.

[75] L. Malaquin, C. Vieu, in Alternative Lithography (Ed: C. M. Sotomayor-Torres) Kluwer Academic/Plenum, New York 2003, pp.169203.

[76] C. Pina-Hernandez, J.-S. Kim, L. J. Guo, P.-F. Fu, Adv. Mater. 2006, in press.

[77] M. D. Dickey, R. L. Burns, E. K. Kim, S. C. Johnson, N. A. Stacey, C. G. Willson, AIChE J. 2005, 51, 2547.

[78] M. D. Dickey, C. G. Willson, AIChE J. 2006, 52, 777.

[79] E. K. Kim, M. D. Stewart, K. Wu, F. L. Palmieri, M. D. Dickey, J. G. Ekerdt, C. G. Willson, J. Vac. Sci. Technol. B 2005, 23, 2967.

[80] E. Mele, F. Di Benedetto, L. Persano, R. Cingolani, D. Pisignano, Nano Lett. 2005, 5, 1915.

[81] D. Y. Khang, H. Yoon, H. H. Lee, Adv. Mater. 2001, 13, 749

[82] M. S. Kim, J. S. Kim, J. Cho, M. Shtein, L. J. Guo, J. Kim, unpublished.

[83] T. Nielsen, D. Nilsson, F. Bundgaard, P. Shi, P. Szabo, O. Geschke, A. Kristensen, J. Vac. Sci. Technol. B 2004, 22, 1770.

[84] L. J. Cheng, M. T. Kao, E. Meyhçfer, L. J. Guo, Small 2005, 1, 409.

[85] Y. Igaku, S. Matsui, H. Ishigaki, J. Fujita, M. Ishida, Y. Ochiai, H. Namatsu, M. Komuro, H. Hiroshima, Jpn. J. Appl. Phys. Part 1 2002, 41, 4198.

[86] S. Matsui, Y. Igaku, H. Ishigaki, J. Fujita, M. Ishida, Y. Ochiai, H. Namatsu, M. Komuro, J. Vac. Sci. Technol. B 2003, 21, 688.

[87] L. Cheng, S. Chang, L. J. Guo, in Proc. MicroTAS 2005, Transducer Research Foundation, Boston, MA 2005, 518. 
[88] Y. Hirai, M. Fujiwara, T. Okuno, Y. Tanaka, M. Endo, S. Irie, K. Nakagawa, M. Sasago, J. Vac. Sci. Technol. B 2001, 19, 2811.

[89] Y. Hirai, T. Konishi, T. Yoshikawa, S. Yoshida, J. Vac. Sci. Technol. B 2004, 22, 3288.

[90] H. D. Rowland, W. P. King, J. Micromech. Microeng. 2004, 14, 1625.

[91] H. D. Rowland, A. C. Sun, P. R. Schunk, W. P. King, J. Micromech. Microeng. 2005, 15, 2414.

[92] H. D. Rowland, W. P. King, A. C. Sun, P. R. Schunk, J. Vac. Sci. Technol. B 2005, 23, 2958.

[93] C. Y. Chao, W. Fung, L. J. Guo, IEEE J. Sel. Top. Quantum Electron. 2006, 12, 134.

[94] S. Ashkenazi, C. Y. Chao, L. J. Guo, M. O'Donnell, Appl. Phys. Lett. 2004, 85, 5418.

[95] Z. N. Yu, H. Gao, S. Y. Chou, Appl. Phys. Lett. 2004, 85, 4166.

[96] H. Tan, A. Gilbertson, S. Y. Chou, J. Vac. Sci. Technol. B 1998, 16, 3926.

[97] S. V. Sreenivasan, A. McMackin, F. Xu, D. Wang, N. Stacey, Micro $\mathbf{2 0 0 5}, 23,37$.

[98] T. Borzenko, M. Tormen, G. Schmidt, L. W. Molenkamp, H. Janssen, Appl. Phys. Lett. 2001, 79, 2246.
[99] L.-R. Bao, X. Cheng, X. D. Huang, L. J. Guo, S. W. Pang, A. F. Yee, J. Vac. Sci. Technol. B 2002, 20, 2881.

[100] N. Kehagias, M. Zelsmann, C. M. S. Torres, K. Pfeiffer, G. Ahrens, G. Gruetzner, J. Vac. Sci. Technol. B 2005, 23, 2954.

[101] Y. P. Kong, H. Y. Low, S. W. Pang, A. F. Yee, J. Vac. Sci. Technol. B 2004, 22, 3251.

[102] X. D. Huang, L. R. Bao, X. Cheng, L. J. Guo, S. W. Pang, A. F. Yee, J. Vac. Sci. Technol. B 2002, 20, 2872.

[103] L. Tan, Y. P. Kong, S. W. Pang, A. F. Yee, J. Vac. Sci. Technol. B 2004, 22, 2486.

[104] D. Suh, J. Rhee, H. H. Lee, Nanotechnology 2004, 15, 1103.

[105] H. C. Scheer, H. Schulz, Microelectron. Eng. 2001, 56, 311.

[106] K. Pfeiffer, A. Fink, G. Gruetzner, G. Bleidiessel, H. Schulz, H. Scheer, Microelectron. Eng. 2001, 57-8, 381.

[107] a) X. Cheng, L. J. Guo, Microelectron. Eng. 2004, 71, 288. b) X. Cheng, L. J. Guo, Microelectron. Eng. 2004, 71, 277.

[108] X. Cheng, D. W. Li, L. J. Guo, Nanotechnology 2006, 17, 927. 